\title{
Two-dimensional numerical study on the effect of water depth on resonance behaviour of the fluid trapped between two side-by-side bodies
}

\author{
Nima Moradi, Tongming Zhou and Liang Cheng* \\ School of Civil, Environmental and Mining Engineering, The University of Western Australia, \\ 35 Stirling Highway, Crawley, WA 6009, Australia \\ *corresponding author: liang.cheng@uwa.edu.au
}

\begin{abstract}
The effect of water depth on gap resonance of two side-by-side rectangular boxes is examined in a 2D numerical wave flume at various gap widths and body drafts. The numerical model used is based on the volume of fluid (VOF) method for the solution of Navier-Stokes equations combined with free surfaces. The water depth covered ranges from shallow to deep water regimes. It is found that the resonant frequencies and wave heights show apparent dependence on water depth. For a given body draft and gap width, the resonant wave frequency shifts to higher values whereas the resonant wave height inside the gap decreases as water depth increases. The amount of reduction of wave height is found to be dependent on the depth to draft and gap width to breadth ratio. It is observed that the effect of water depth on resonance frequency is very small when the ratio of water depth to body draft is equal to or greater than six, while the effect of water depth on resonant water level diminishes when this ratio increases beyond 10. Furthermore, it is observed that the variation trends of the resonant frequency and the reduction of the resonant wave height with water depth are similar at different drafts and gap widths. The present results suggest that the water depth, body draft and the ratio of water depth to body draft are the key factors that influence the resonant responses. It is also found that the potential flow model not only over-predicts the resonant wave heights but also fails to predict the variation trends of the resonant wave height with water depth.
\end{abstract}




\section{INTRODUCTION}

Nowadays as the oil and gas exploration and production have moved to deep and ultra-deep waters, floating liquefied natural gas (FLNG) facilities show great potential as the most economic ways to process and distribute the hydrocarbon products. One of the key technical challenges for FLNG facilities is the safety of offloading operations under harsh environmental conditions. The preferred offloading option for FLNG systems appears to be the side-by-side offloading where the LNG carrier is positioned side-by-side with the FLNG platform. When two floating bodies are arranged side-by-side in close proximity, fluid resonance inside the narrow gap between the vessels becomes a concern because it may induce large amplitudes of response. Therefore, accurate prediction of the resonance behaviour is crucial in evaluating the operability of offloading.

Many researchers have studied the hydrodynamic interactions of multiple bodies with narrow gaps in between. The methods used in these investigations include theoretical analysis, physical model testing and numerical simulation. So far, potential flow models have been used more than any of the other methods to deal with this problem (Miao et al. [1], [2]; Li et al. [3], Zhu et al. [4], Sun et al. [5]). However, it has been understood that while the potential flow theory is capable of predicting the resonant wave frequency successfully, it overpredicts the resonant wave height in the gap near the resonant frequency and consequently leads to over-estimation of the wave forces on the floating bodies. Different methods have been introduced in order to achieve more realistic predictions of the water elevation inside the gap. For example, Buchner et al. [6] and Newman [7] added lids on the gap free surface; Chen [8] introduced a linear dissipative term in the free surface boundary condition of the potential flow model. In addition, Molin et al. [9] added a massless lid on the free surface,

which was assigned a damping force quadratic with respect to the vertical velocity. The 
method of Chen [8] has been used by Pauw et al. [10], Bunnik et al. [11] and Lu et al. [12]. Satisfactory results have been obtained using the proposed methods that lead to a more accurate estimation of the resonant wave height in the gap. However, the level of damping in these studies must be tuned using pervious data such as model test results. In addition to modifying the surface boundary condition, Chen et al. [13] utilised a quadratic pressure loss boundary condition in the suitable position inside the fluid domain in order to introduce the energy dissipation. The effect is similar to placing a physical perforated plate in the fluid domain.

Computational fluid dynamics (CFD) has also been used to investigate the fluid resonance in the narrow gap. Reported results (e.g. Lu et al. [14], [15], [16], [12]; Sauder et al. [17], Moradi et al. [18]) have shown satisfactory agreement with those obtained from experiments, indicating that the viscous fluid model performs well in predicting the violent free surface oscillation at the fluid resonance. The effects of different parameters, such as body draft, gap width and body breadth on resonant characteristics in side-by-side offloading operations were also studied by Saitoh e al. [19], Sun et al. [5], Lu et al. [12] and Liu and Li [20]. Furthermore, Kristiansen and Faltinsen [21] studied the resonant water motion between a ship and a terminal in shallow water. Comparison of their numerical simulation results with that obtained from the model tests showed that the terminal gap kinematics is over predicted by the nonlinear and especially the linear potential theories around the resonance frequency. While the behaviour of the linear theory was expected, that of the nonlinear theory was not a priori known.

Moradi et al. [18] showed that a curved corner setup at the gap entrance resulted in an increase in the resonance wave height and a decrease in the resonant frequency for different 
corner roundness ratios. The present study will examine the effect of water depth on gap resonance characteristics for bodies with sharp corner inlet and compare the results with those reported previously using both experimental and numerical methods. The detailed effect of the corner shapes and gap inlet configurations on the flow near the corners and inside the small gap, as well as the gap resonance characteristics can be found in [18].

It is noted that, to the authors' knowledge, none of the previous studies have considered the influence of water depth regimes on the resonance characteristics. Most of them have mainly reported findings in the transitional water depths. It is anticipated that in the interest of evaluating the response of multiple bodies in close proximity more accurately, additional aspects such as varying water depth, which have not yet been explicitly investigated, need to be considered. In this context, the primary aim of the present work is to explore the effect of the offloading operation water depth on the variation of the characteristics of the gap resonance using computational fluid dynamics method. The numerical model used in this study is built on the OpenFOAM platform and implemented on a massive parallel computing facility hosted by the Pawsey supercomputing centre in Western Australia. This paper is organised as follows. After a brief introduction in Section 1, the background of the numerical model, the numerical setup and the wave parameters used in the simulations are described in Section 2. Simulation results of the dependency of the gap resonant wave height and frequency on water depth, body draft and gap width as well as variation of forces acting on the bodies in the different water depths are shown in Section 3. Comparisons between results of present numerical simulation and the potential flow model for different water depths are also conducted in this part. Finally, the conclusions are drawn in Section 4. 


\section{NUMERICAL WAVE FLUME}

\subsection{Numerical model setup and boundary conditions}

In the present study the numerical wave tank is $18.5 \mathrm{~m}$ (length) $\times 0.1 \mathrm{~m}$ (width), and the height is changing between $1.0 \mathrm{~m}$ to $5.0 \mathrm{~m}$ based on the desired water depth for different simulations. Figure 1(a) shows the simulation domain and the definition of the co-ordinate system which is defined in such a way that the origin is located at the seabed and $x$ is in the wave propagation direction and $y$ is in the upward direction. The thickness of the domain in $z$-direction corresponds to one cell. Two rectangular bodies with sharp corners are used to represent the ships which are located at the middle of the simulation domain. The regular incident wave propagates with an incident angle of $90^{\circ}$ relative to the ship length. Two rectangular relaxation zones of $5 \mathrm{~m}$ long each are implemented at the inlet and outlet boundaries of the simulation domain to avoid reflection of waves from the outlet boundary. The absence of a method to suppress the internal wave reflections in the computational domain can lead to contaminated results and create discontinuities in the surface elevation at the wave making boundary. It should be noted that the length of the relaxation zones are kept constant for different wave frequencies covered in this study. Utilizing the current relaxation technique, Jacobsen et al. [22] showed that for relaxation zones equal or larger than the wave length, the reflection effect is minimum. Similarly, our simulations show that the outlet boundary is qualitatively non-reflective (results are not given here). Further details of the relaxation technique used in this study can be found in [22]. The ship beam (breadth), draft, gap width and water depth are defined by $B, d, B_{g}$ and $h$, respectively, as illustrated in Figure 1(b). The ship beam, which is $0.5 \mathrm{~m}$ in all simulations, is the same for both bodies, and the corresponding values for the other three parameters are changing in accordance with the simulation requirements. These values are tabulated in Tables 1 and 2 . The bodies have a 
bottom clearance of $h-d$. The incoming wave height $H_{0}$ is fixed at $0.024 \mathrm{~m}$ and the wave length $L$ varies between $1 \mathrm{~m}$ and $6.28 \mathrm{~m}$. These values are similar to those used in Saitoh et al. [19], Lu et al. [15] and Moradi et al. [18].

At the bottom of the wave tank and the solid walls of the bodies, no slip boundary condition is applied. Zero-gradient is applied for outflow (as defined by the flux) and for inflow the velocity is obtained from the patch-face normal component of the internal-cell value. In order to represent the atmospheric condition for the upper boundary of the wave tank, the velocity is set to the "pressureInletOutletVelocity", a complex boundary condition defined in OpenFOAM, which prescribes Neuman or Dirichlet types of boundary conditions on the pressure and velocity depending on the direction of the velocity vector. The pressure condition for the inlet boundary is set to zero gradient and the velocity is defined as the incoming wave velocity. The numerical domain used in this study is meshed utilising OpenFOAM mesh generation capabilities. First a structured background mesh is generated using the "blockMesh" utility in such a way that the cell size decreases from both the inlet and the outlet boundaries towards the centre of the simulation domain. The same approach is applied in the vertical direction. The next step is to generate the boundaries of the two floating bodies by subtracting the desired volumes from the background mesh using the "subsetMesh" utility. The outcome will be a rectangular simulation domain with higher mesh density around the bodies (as shown in Figure 2). In case of deep and ultra-deep water simulations the mesh density inside the domain is kept constant by multiplying the number of cells in the vertical direction by the depth ratio which is defined as the new depth over initial depth of $0.5 \mathrm{~m}$. It should be noted that extensive checks for the dependency of the simulation results on mesh density have already been conducted for water depth $h=0.5 \mathrm{~m}$ in [18] and the corresponding higher water depths in this paper are modelled based on their work. Simulations start from still water state and run for about 50 seconds to ensure the steady state 
condition is reached. In order to generate different types of waves, a separate file containing different wave properties should be located in the "constant" directory of the simulation folder. For the case of linear wave generation, water depth, wave height and period will be required to calculate the other parameters such as wave number and frequency.

In order to check the proper functionality of the wave generating boundary, Figure 3 shows the time series of wave elevation for the shortest and longest waves at $x=6 \mathrm{~m}$, i.e. $1 \mathrm{~m}$ downstream of the inlet relaxation zone. The results illustrate regular variation of the water elevation for the 50 seconds of simulations, which corresponds to 62.5 and 24.2 periods in the two studied cases, indicating satisfactory performance of the inlet boundary in generating regular waves.

\subsection{Formulation and solution of the problem}

The problem is analysed with a Navier-Stokes equations solver based on the OpenFOAM libraries version 2.1.x. The two-phase solver "waveFoam" is used for this study, which allows for simulations of wave-structure interactions in OpenFOAM based on the finite volume method (FVM) by assuming incompressibility and immiscibility of air and water. WaveFoam incorporates the wave generation and absorption mechanisms with the existing OpenFOAM two phase flow solver "interFoam". Detailed explanation on how the solver is developed and how the wave generation and absorption methods are implemented in the code can be found in Jacobsen et al. [22]. The interface between the two phases is captured by a volume of fluid (VOF) approach, for instance, as presented in Hirt and Nichols [24]. The governing equations of motion of an incompressible fluid are

$$
\partial_{t} u_{i}+u_{j} \partial_{j} u_{i}=-1 / \rho \partial_{i} p+v \nabla^{2} u_{i}+E_{i} \pm \sigma_{t} k_{\gamma} \nabla \gamma
$$

and

$$
\partial_{j} u_{j}=0,
$$


where $u_{i}$ are the velocity components, $p$ is the pressure, $\rho$ is the density, $E_{i}$ are the components of the external forces per unit mass, $v$ is the kinematic viscosity of the fluid, $t$ is the time, and the indices $i$ and $j$ refer to the space coordinates $x_{i}$ and $x_{j},(i, j=1,2,3)$. The partial differentiations $\partial_{i}$ and $\partial_{t}$ denote derivatives with respect to $x_{i}$ and the time t, respectively. The last term in Eq. (1) is the surface tension term, where $\sigma_{t}$ is the surface tension coefficient and $k_{\gamma}$ is the surface curvature. The surface tension coefficient between air and ocean water is considered as $0.07 \mathrm{~m} / \mathrm{s}^{2}$. It should be pointed out that no turbulent model is included in the present viscous numerical wave flume. Instead, direct numerical simulations are applied in the gap region and around the bodies with very fine meshes

The above equations are solved for both air and water simultaneously. The fluid is tracked using a scalar field $\gamma$ which takes a value of 0 for air and 1 for water and intermediate values for a mixture of air and water. In the computational cells adjacent to a boundary, the common face between the cell and the boundary (boundary face) can have three different states of fully wet (completely submerged), fully dry (completely above water) and intersected by the water surface. For the dry faces the boundary conditions are:

$$
n \nabla p=0, \quad u=0, \quad \gamma=0,
$$

where $\mathbf{n}$ is the boundary unit normal vector. The boundary conditions for the wet faces are given analytically according to the chosen potential wave theory (Linear wave theory in this case), which is evaluated at the face centres. For the boundary faces intersected by the free surface, a local linear approximation of the real surface, $\eta^{*}$, is defined based on two intersection points. It forms a closed and wet polygon together with the wet sides of the boundary face. The corresponding wet area, $A_{W}$ and wet centre $C_{W}$, can be computed using simple geometrical methods. On the basis of the sub-division of the boundary face, $\gamma$ at the boundary is specified as $A_{W} / A_{F}$, where $A_{F}$ is the area of the boundary face. $\mathbf{u}$ and $n \nabla p$ are 
evaluated from the potential flow theory at $C_{W}$ and applied to the boundary face. This approach, which was first introduced by Jacobson et al. [22] as a part of their wave generation toolbox, is favoured to the typical method, where the face is either wet or dry based solely on the location of the face centre relative to the free surface, as the latter approach resulted in unrealistic oscillations on the water surface. By applying the new approach, the fake fluctuations are avoided due to the smoothness introduced at the interface.

The distribution of $\gamma$ is modeled by an advection equation

$$
\frac{\partial \gamma}{\partial t}+\nabla \cdot[\mathrm{u} \gamma]+\nabla \cdot\left[u_{r} \gamma(1-\gamma)\right]=0
$$

The last term on the left hand side of Eq. (4) is a compression term, which limits the smearing of the interface, and $u_{r}$ is the relative velocity of the phases. The method is developed by OpenCFD® and documented in [25].

Using $\gamma$ one can express the spatial variation of any fluid property $\emptyset$ through the weighting as shown in Eq. (5),

$$
\emptyset=\gamma \emptyset_{\text {water }}+(1-\gamma) \emptyset_{\text {air }}
$$

The exponential relaxation function as shown in Eq. (6) is the preferred relaxation technique used in this study

$$
\alpha_{R}\left(\chi_{R}\right)=1-\frac{\exp \left(\chi_{R}^{3.5}\right)-1}{\exp (1)-1} \text { for } \chi_{R} \in[0 ; 1]
$$

This is applied inside the relaxation zone in the following way

$$
K=\alpha_{R} K_{\text {computed }}+\left(1-\alpha_{R}\right) K_{\text {target }},
$$


where $K$ represents either velocity $(\mathrm{u})$ or volume of fraction of water/air $(\gamma) . \alpha_{R}$ is always equal to 1 at the interface between non-relaxed part of the computational domain and the relaxation zone.

\section{RESULTS AND DISCUSSION}

\subsection{Dependency of resonant characteristics on water depth}

So far, most of the existing numerical simulations and experiments are conducted for single fixed water depth which mainly falls within the transitional depth regime. Whereas in reality the operations may take place in different depth regimes, especially nowadays when the explorations are conducted in deep and ultra-deep waters. In order to explore the dependence of gap resonant characteristics on water depth, simulations are carried out in various water depths, ranging from $h=0.3 \mathrm{~m}$ up to $h=3 \mathrm{~m}$ for three different body draft $d$ and five $B_{g}$ values for bodies with a fixed width of $B=0.5 \mathrm{~m}$. The water depth regimes correspond to shallow $(h=0.3 \mathrm{~m}$ and $0.4 \mathrm{~m})$, transitional $(h=0.5 \mathrm{~m}, 0.75 \mathrm{~m})$ and deep water $(h=1 \mathrm{~m}, 1.5 \mathrm{~m}$, $2 \mathrm{~m}$ and $3 \mathrm{~m}$ ) with low, medium and high bottom clearances respectively. The initial results for the evolution of resonant wave height and frequency as a function of water depth are compared with those obtained using HydroSTAR (HydroSTAR For Experts Version 7.03), which is a hydrodynamic software developed by Bureau Veritas as a diffraction/radiation software based on potential flow theory. It takes into account the multi-body interactions, the effects of forward speed and internal liquid motions. More information about this software can be found in [23]. Later on the numerical results based on viscous flow are compared with those obtained in studies with a similar setup and a constant water depth within the transitional water depth regime $(h=0.5 \mathrm{~m})$ (Lu et al. [12], Moradi et al. [18], Saitoh et al. [19]). The influence of water depth on resonant wave height and frequency is examined and 
compared between different cases. Tables $1 \& 2$ list the numerical values of the different parameters used to simulate 30 different cases and show how the simulations are grouped into two sub-sections based on coupled variation of two of the main four influential parameters.

Figure 4 shows the dependency of the resonant characteristics on water depth when the bodies have a fixed draft of $d=0.25 \mathrm{~m}$ and constant gap width of $B_{g}=0.05 \mathrm{~m}$. The results for nine different depths ranging from $0.3 \mathrm{~m}$ to $3 \mathrm{~m}$ are shown in two separate figures. Figure $4 \mathrm{a}$ is for shallow and transitional water regimes and Figure $4 \mathrm{~b}$ is for deep water regime. What is more, one case for deep water regime $(h=1 \mathrm{~m})$ is also included in Figure $4 \mathrm{a}$ so that the differences between different water depth regimes can be seen clearly. The present results at water depth $h=0.5 \mathrm{~m}$ agree well with those reported previously in [12], [18] and [20], i.e. they all peak at about $\omega /(\mathrm{g} / \mathrm{B})^{0.5}=1.2$ with wave heights $H_{g} / H_{0}=4.3 \sim 5$, although the results from the mentioned studies are not shown. It can be seen that increasing water depth, i.e. migrating from shallow to transitional and deep water regimes, has considerable effects on both the resonant frequency and the wave height inside the gap. With the increase of water depth, the resonant frequency shifts to higher values monotonically until deep water regime is achieved, where the resonance frequency does not change anymore. In contrast, the resonant wave height decreases monotonically with the increase of water depth, albeit at different rate for shallow, transitional and deep water regimes. It is pertinent to examine the dependence of resonance frequency and wave height on water depth predicted using potential flow theory and compare with the results obtained for viscous flows. Figure 5(a-b) shows the numerical results based on a potential flow model (Hydrostar 7.03) for the variation of the nondimensional wave height inside the gap of two rectangular boxes as a function of nondimensional incident wave frequency for the setups similar to those used in viscous models (results are shown in Figure 4) with $d=0.25 \mathrm{~m}$ and $B_{g}=0.05 \mathrm{~m}$. It can be seen from 
Figure 5 that the potential flow method predicts the same trend of variation of the resonant wave frequency with water depth as that from the viscous model. However, the two models predict different trends of variation for the resonant wave height with water depth. The viscous flow calculations suggest that the resonant wave height decreases with the increase of the water depth up to a depth (deep water regime as shown in Figure 4b) where further increase in water depth will not affect the resonant wave height significantly. However, the calculations based on potential flow display an opposite trend. The capability of the inviscid flow theory in predicting the resonant frequency is a priori known. It has also been shown in many previous studies that the potential flow theory over-predicts the resonant wave height inside the narrow gap.

The increase in the resonant frequency predicted by both the viscous and inviscid models is anticipated and can be explained by Eq. (8) which was theoretically derived by Saitoh et al. [19] for calculation of the resonant wave frequency in a rectangular wave tank between twin rectangular bodies.

$$
\omega_{g}=\sqrt{\frac{g}{\frac{B^{B} B}{h-d}+d}} .
$$

According to Eq. (8), the resonant frequency is directly related to the water depth, i.e. increasing water depth $(h)$ will increase the resonant frequency. The same trend is apparent in Fig. 4. However, when the water depth is more than half of the wave length $(h / L>0.5)$, i.e. in the deep water regime, the effect of water depth on resonance frequency is much less than that in shallow and transitional regimes and can be neglected. This is also consistent with the theoretical fluid resonance appearance condition in the gap as shown in Eq. (9) derived by Saitoh et al. [19]. In their study they have concluded that in case of deep water, Eq. (9) can be rewritten as Eq. (10). 
$\frac{l}{\lambda}=\frac{1}{2 \pi}$

where $l=\frac{B_{g} B}{h-d}+d$, is the geometrical condition. Furthermore, Kristiansen and Faltinsen [21] have reported reduction in the resonance wave height with the increase of water depth based on the time history measurements of their shallow water tests of $h=0.29 \mathrm{~m}$ and $h=0.4 \mathrm{~m}$ depths.

It is arguable that the discrepancy in the trend for the change of resonant wave height as a function of water depth between the viscous model and the potential flow model can be explained in the following ways; In shallow and transitional water regions $(h=0.3 \mathrm{~m}, 0.4 \mathrm{~m}$ $0.5 \mathrm{~m}$ and $0.75 \mathrm{~m}$ ) the wave group velocity (celerity) is approximated by $\sqrt{g h}$, in which $g$ is the gravity acceleration and $h$ represents the water depth. Based on this approximation increasing the water depth will increase the wave group velocity, i.e. the speed at which the wave spectrum energy is transferred. However as the potential flow theory does not consider any energy dissipation due to flow separation and viscous effects, higher wave group velocities will result in higher amount of energy being transferred into the gap region and generate higher water elevations. While for real fluid, it is anticipated that the increase in the flow velocity will increase the amount of energy dissipation due to viscous dissipative effects as well as flow separation and generation of flow recirculation regions near the sharp corners of the bodies. Furthermore, it is also believed that the decrease of the resonance wave height in the gap by the increase in water depth lies in the fact that, although the amount of water trapped inside the gap is unchanged in different water depths, there would be significant change in the area under the floating bodies and so the fluid bulk. This means that the inertial mass will increase and the greater mass will result in a greater tendency to resist the velocity 
change with the same amount of depth independent external force. It has been checked that increasing water depth will lead to reduction of the fluid velocity entering the gap. The velocity variations at the water surface in the middle of the narrow gap for the frequency corresponding to the resonance frequency of the three different investigated water depths (same frequencies as shown in Figure 4) are shown in Figure 6. As expected, it is noticeable that the highest velocity magnitude occurs at the lowest water depth studied here (i.e. $h=0.5$ m) and it decreases with the increase in water depth. This leads to the subsequent reduction of the resonant wave height inside the gap, which is consistent with the results shown by Lu et al. [16]. Generally for deep waters, two factors contribute to the level of gap wave height. One is the increased level of inertia due to increased fluid bulk under the bodies and the other is the reduction of flow velocity. Although the reduced velocity should technically reduce the viscous dissipation and increase the wave height, the inertial force seems to be the dominant factor in this case. In addition, for viscous flows, the present results agree well with that obtained by Lu et al. [15], which showed that the non-dimensional wave height is analogues to the non-dimensional incident vertical velocity component.

The numerical investigations show that the magnitude of the pressure forces acting on the bodies is closely correlated to the water depth. The time-series of the horizontal forces on the front body for a given frequency and at the resonant frequencies for different water depths are shown in Figures 7 and 8, respectively. In these figures the vertical axis represents the force normalized by $\rho g A_{0} B$, where $A_{0}$ is the incident wave amplitude. It can be seen from these figures that the magnitude of the horizontal force in shallow water is much larger than that in intermediate and deep water regimes. The amount of this difference increases at the resonance frequency where the horizontal force magnitude for $h=0.3 \mathrm{~m}$ is about three times of that for $h=2 \mathrm{~m}$. The variations of the magnitude of the horizontal and vertical forces on 
the front body with respect to wave frequency at different water depths are shown in Figures 9 and 10, respectively. It can be seen from Figure 9 that the magnitude of the horizontal force in shallow water $(h=0.3 \mathrm{~m})$ is the largest and it decreases with the increase of water depth. The trends for the variation of the force magnitudes are analogous to that of the evolution of the wave height as a function of water depth. Lu et al. [15] also observed a similar phenomenon in the study of forces acting on three side-by-side bodies with two gaps in between. They showed that the variation of the horizontal force amplitude as a function of frequency is similar to that of the wave height amplitude. The dependence of the vertical force magnitude on wave frequency for different water depths is examined in Figure 10. It should be noted that the hydrostatic pressure of the force is excluded from the calculations shown in Figure 10. The figure demonstrates that the variation of the vertical force magnitude by frequency for a given water depth is small (within about 5\%) and it can be considered as constant. While the values of the vertical force magnitude are approximately the same for intermediate and deep water regimes $(\mathrm{h}=0.5 \mathrm{~m}, 1 \mathrm{~m}$ and $2 \mathrm{~m})$, they are only about half of those in shallow water $(\mathrm{h}=0.3 \mathrm{~m})$. The inset highlights the variation of the vertical force with frequency in transitional and deep water regimes.

It should be noted that the resonant wave height and frequency are deemed not only to be dependent on water depth, but also on body draft and the ratio of water depth to body draft. This is presented in detail in the next section where discussion is focused on how resonant characteristics are affected by water depth and body draft couplings.

\subsection{Influence of the water depth and body draft couplings on resonant characteristics}

In order to examine the effect of water depth on gap resonance at different body drafts, simulations are conducted at three different body drafts and five different water depths which 
totally blend 15 different cases as listed in Table 1. For all of these simulations with varying drafts and water depths, the gap width is constant value of $0.05 \mathrm{~m}$. The effect of body drafts at various water depths are then investigated by comparing the results for fixed draft values in different water depths as well as different drafts in a fixed water depth. This gives a good insight on all the possible coupling scenarios when these two parameters are studied together.

Figure 11 illustrates the variation of the non-dimensional wave height as a function of wave frequency at different drafts. The water depth is the same for all simulations and is equal to 1 $\mathrm{m}$, which corresponds to the deep water regime. The results show that increasing $d / h$ or just the draft in case of a fixed water depth will result in the reduction of the resonant frequency, which is already explained theoretically in [20]. However, at the same time the resonant wave height inside the gap increases sharply in such a way that the resonant wave height for $d / h=0.45$ is almost twice as that for $d / h=0.125$. When the draft to depth ratio increases, the bottom clearance becomes smaller and the fluid under the bodies will flow into a region similar to a channel. It is speculated that the higher wave heights at higher draft to depth ratios are associated to the reduction in the area under the bodies and the subsequent increase in the fluid velocity for the same wave condition. This phenomenon may also be explained by the fact that the same amount of external force is acting on less amount of fluid bulk mass underneath the bodies as justified earlier for the water depth dependency. Further examination of Figure 11 brings about the question whether two bodies with the same $d / h$ ratio but different body draft at two different water depths would display similar performance. In order to answer this question, two different cases with different drafts and water depths are compared to each other, with first case having body draft and water depth values as $d=0.125$ and $h=1 \mathrm{~m}$ respectively and the second case with both of these values doubled, i.e. keeping the $d / h$ ratio constant at 0.125 . As shown in Figure 12, despite 
having the same draft to depth ratio, there are significant differences in both resonant wave height and frequency between the two cases. When water depth $h$ increases, the nondimensional resonant frequency reduces from 1.6 to 1.2 , whereas the non-dimensional resonant wave height increases from 2.2 to around 3.3. Figure 12 suggests that each of these two individual parameters $(h \& d)$ is important when studying resonant characteristics of fluid trapped in the narrow gap. In addition, two other cases with the same draft but two different water depths are compared to each other and shown in Figure 13. It is observed that despite having different water depths, the resonant wave height and frequency coincide very well between the two cases. It needs to be noted that although water depth was previously demonstrated to be an important factor for resonant behavior, its effect is mainly noticeable when a change of water depth regime occurs, similar to what is shown in Figure 4.

Figure 14(a-c) illustrates the evolution of the resonant wave height as a function of incident wave frequency. The results are shown for five different water depths which correspond to transitional and deep water regimes and for three different body drafts, covering cases 1 to 15 as listed in Table 1. It is observed that regardless of the draft value, the trends are generally the same, i.e. the resonant frequency increases and the wave height decreases with the increase of water depth. However, comparing the results obtained at different body drafts (Figures 14a-c), it can be seen that not only the amount of reduction of the resonance wave height inside the gap, but also the location of the peak (or resonant) frequency depends on both the water depth and body draft. As mentioned earlier, for any given body draft, the increase in water depth leads to an increase in resonant frequency and a reduction in resonant wave height. However, when the body draft increases, the reduction of the resonant wave height in deeper water becomes more significant. For example at $d=0.35 m$ (Figure 14c), the results show that the resonant wave height decreases by approximately $36 \%$ for $h=2 \mathrm{~m}$ 
compared with $h=0.5 \mathrm{~m}$, whereas for $\mathrm{d}=0.15 \mathrm{~m}$ (Figure 14a) the amount of this reduction is about $26 \%$. Although the non-dimensional wave height decreases substantially when the depth regime changes from transitional to deep water, it can be seen that even the reduced deep water $(h=2 \mathrm{~m})$ resonance wave height for $\mathrm{d}=0.35 \mathrm{~m}$ is higher than that for $\mathrm{d}=0.15 \mathrm{~m}$ at the transitional regime $(h=0.5 \mathrm{~m})$. This result clearly shows that while both water depth and body draft have significant influence on resonant wave height, the latter plays a much more important role in defining the magnitude of the non-dimensional wave height inside the gap. The detailed illustrations on the evolution of wave height as a function of the incident wave frequency for each of the five studied water depths covering all of the three different drafts are given in Figure 15(a-e). It can be seen that regardless of the water depth, the resonant frequencies appear to be very close to each other for a given draft whereas the nondimensional wave height in the gap increases linearly as a function of body draft irrespective of the depth regime. It is also notable that the peaks for the maximum non-dimensional wave height for different drafts fall in the same positions relative to each other in different water depths.

Variations of the resonant frequency as a function of water depth normalized by the body draft for three different body drafts are shown in Figure 16. It is observed that for any given draft the resonant frequency increases till it reaches a plateau for water depths equal or greater than six times the body draft where the resonant frequency is no longer influenced by the water depth. Having known that the resonant frequency has an inverse relationship with the body draft, Figure 16 shows that the major difference between the trends for the evolution of the resonance frequency as a function of water depth for different body drafts is the rate at which the depth independency is achieved. While for $\mathrm{d}=0.15 \mathrm{~m}$ the resonant frequency rises sharply till it reaches a constant value, for $\mathrm{d}=0.35 \mathrm{~m}$ the increment slope is much smaller. 
Figures 17 and 18 are shown in order to provide a better understanding of the dependency of the resonance wave height and frequency on water depth. The variations of the maximum wave heights at resonant frequency (extracted from Figure 14) as a function of water depth for three different body drafts are shown in Figure 17. It can be seen that migrating from transitional to deep water regimes tends to gradually decrease the wave height irrespective of the draft. For the three draft values studied, the slopes of the wave height curves decrease as $h / d$ increases till it finally becomes small at about $h / d=10$, i.e. if water depth reaches a value equal to or greater than 10 times the body draft, it would be possible to neglect the effect of water depth on resonant wave height in the gap. For the water depth where transition between the two different depth regimes takes place $(h=1 \mathrm{~m})$, different locations can be distinguished as the critical depths for each body draft (indicated by arrows in the figure). Figure 18 compares the variation of the wave height as a function of water depth normalized by body draft at different water depths. It can be seen that for all water depths, the magnitudes of $H_{g} / H_{0}$ decrease sharply initially with the increase of $h / d$, especially for the transitional water regime $(\mathrm{h} \leq 1)$. Although very large values of $h / d$ are not examined in this study, it is speculated that as $h / d$ goes to infinity the slope of the figure gradually decreases and the variation of non-dimensional wave heights as a function of water depth approach to constant values with magnitudes depending on water depth.

The sole effect of body draft on wave height has been extensively studied before in $[12,18$, and 19] and the results showed that the tendency for the wave height to increase with the draft is mainly related to the wave conditions under resonance (wave frequency and length). As shown earlier, the resonant frequency is lower for higher drafts due to the expansion of the water volume and the increase of the mass inside the gap. Lower resonant frequency means 
longer waves and less reflection by the bodies, which leads to less energy dissipation and higher wave amplitudes. However, the reason that the amount of this increase in wave height is less in deeper waters than that in transitional waters is mainly due to the higher mass of fluid underneath the floating bodies, which reduces the slope of the wave height escalation.

\subsection{Influence of the water depth and gap width couplings on resonant characteristics}

In order to examine the coupled effect of gap width and water depth on resonant characteristics, four different gap widths ranging from $0.025 \mathrm{~m}$ to $0.1 \mathrm{~m}$ with an increment of $0.025 \mathrm{~m}$ are studied for each water depth. The dependency of gap resonance on water depth has already been shown in the previous section and in Figure 4 where all the simulations are conducted at the same gap width $\left(B_{g}=0.05 \mathrm{~m}\right.$ ). Fifteen new cases are introduced for three new gap widths as shown in Table 2. The variations of $H_{g} / H_{0}$ with respect to the incident wave frequency for these new cases alongside the existing results for $B_{g}=0.05 \mathrm{~m}$ at five different water depths are shown in Figure 19(a-e). The body draft value for all these simulations is constant and equal to $0.25 \mathrm{~m}$. It can be seen that the overall trend for the variation of the resonant frequency for different gap width is the same, i.e. it decreases with the increase of gap width regardless of the water depth. This is consistent with the predictions based on Eq. (8). This result is also consistent with the result obtained by Moradi et al. [18], where it was shown that the resonant frequency is inversely proportional to $\mathrm{B}_{\mathrm{g}}$ whereas the wave height in the gap depends apparently on $\mathrm{B}_{\mathrm{g}}$, with critical gap width being dependent on the setup (body draft and other geometric parameters such as the ratio of gap width to body breadth). They also showed that the wave height inside the gap directly relates to the vertical velocity of water in the gap. Initially, the flow velocity entering the gap increases with the increase of the gap width. However, as the gap width exceeds a critical value, the frictional 
resistance dominates the fluid motion, resulting in a reduction of flow velocity and subsequent wave height [12].

For convenience in examining the variation of the resonance frequency with gap width, Figure 20 re-plots the resonance frequency as a function of gap width for five different water depths covered in this study. It can be seen that although the resonant frequency generally decreases with the increase of gap width, the reduction rate is much more significant in the transitional depth regime $(h=0.5 \mathrm{~m}, 0.75 \mathrm{~m})$ than that in the deep water regime $(h=1 \mathrm{~m}$, $1.5 m$ and $2 m$ ). In addition, it can be observed that the dependence of the resonant frequency on gap width in deep water regime overlap each other, emphasizing the non-sensitivity of the resonant frequency to water depth in deep waters as earlier seen in Figure 4. Similarly, for convenience to examine the variation of the resonance wave height with gap width, Figure 21 re-plots the maximum resonant wave heights as a function of gap width for five different water depths. The common behaviour of $\mathrm{H}_{\mathrm{g}} / \mathrm{H}_{0}$ among different water depths is found when $B_{g} / B<0.15$, where the wave height increases with the increase of the gap width. It is also evident that for a given gap ratio $\mathrm{B} / \mathrm{B}, \mathrm{H}_{\mathrm{g}} / \mathrm{H}_{0}$ decreases with the increase of water depth. When the gap ratio is large enough, say 0.2 , in deep water, $\mathrm{H}_{\mathrm{g}} / \mathrm{H}_{0}$ does not depend on water depth anymore. This is not the case for the transitional water depths.

Figure 22 demonstrates the evolution of the wave height as a function of wave frequency at all 5 different water depths for 4 different gap widths. It can be seen that for $\mathrm{B}_{\mathrm{g}}=0.05 \mathrm{~m}$, $0.075 \mathrm{~m}$ and $0.1 \mathrm{~m}$, the trends look similar, i.e. resonance frequency increases with increasing $\mathrm{h}$, while for $\mathrm{B}_{\mathrm{g}}=0.025 \mathrm{~m}$, the resonance frequency is roughly the same for different $h$. The difference between the resonance frequencies recorded for the highest and the lowest water depth increases with the increase of gap width, changing from nearly zero for $\mathrm{B}_{\mathrm{g}}=0.025 \mathrm{~m}$ to 
about 0.2 for $\mathrm{B}_{\mathrm{g}}=0.1 \mathrm{~m}$. It is also observed that disregarding the water depth, resonance frequency decreases with $B_{g}$, which is again consistent with Eq. (8).

Figure 23 summarises the variations of the wave height as a function of normalized water depth for different gap widths. It can be seen that the magnitude of wave height depends on both water depth and gap width and the trends for different gap width are similar to those previously seen in Figure 17 for the dependency of wave height on the coupled draft and depth parameters. For the water depth covered in this study, the strongest dependency on water depth is found for $B_{g}=0.025 \mathrm{~m}$, where the magnitude of $H_{\mathrm{g}} / H_{0}$ decreases by around $45 \%$, changing from 4.68 to 2.57 when the water depth increases from 2 to 8 times the body draft. When $h / d \geq 6$, for all gap widths, it seems that $H_{\mathrm{g}} / H_{0}$ does not depend on water depth anymore. For $B_{g}=0.05 \mathrm{~m}, 0.075 \mathrm{~m}$ and $0.1 \mathrm{~m}$, the amount of wave height reductions due to water depth increase are $29 \%, 28 \%$ and $21 \%$, respectively, indicating that increasing the gap width between the floating bodies will decrease the amount of reduction in the resonant wave height caused by migrating from shallow to deep water regions. While no simulation has been conducted for the gap width within the range of $0.025 m<B_{g}<0.05 m$, according to Figure 23, it is speculated that the non-dimensional wave heights for this water depth at high $h / d$ should be in the range of $2.5-3.5$ and the point where $H_{\mathrm{g}} / H_{0}$ reach a plateau should fall somewhere in the range of $4<h / d<6$. After all, considering all simulation results obtained in this part, it is shown that the resonant wave height is a function of both gap width and water depth and one cannot discuss about the optimum gap width between the bodies for a given setup without referring to water depth regimes. 


\section{CONCLUDING REMARKS}

Navier-Stokes equations are solved to investigate the resonance characteristics in the gap between twin bodies of rectangular sections arranged in close proximity. In order to get a clear understanding of the influence of water depth on resonant frequency and wave height inside the gap, five different water depths are considered at different gap widths and body drafts comprising 30 different cases in total. The simulation results are compared with the existing results obtained at the transitional water depth. The main findings are as follows:

- The present study shows that water depth has a significant influence on resonance behaviour of water trapped in the gap between twin bodies in side-by-side arrangement. For a given body draft and gap width, the resonant wave frequency increases while the wave height decreases with the increase of water depth. The former is due to the direct relation between water depth and resonance frequency which is consistent with the theoretical prediction, whereas the latter is due to the increase of the fluid bulk underneath the floating bodies and subsequent flow velocity reduction.

- It appears that the potential flow model not only over-predict the resonant wave height, but also give an incorrect variation trend of the resonant wave height with water depth.

- When the gap width and body draft are allowed to change, the effect of water depth on gap resonance characteristics will also vary depending on the former two parameters. Considering the influence of water depth and body draft on resonant wave height as coupled parameters, the latter plays a much more important role in defining the magnitude of the non-dimensional wave height inside the gap. 
- When the water depth is more than half the wave length (i.e. in deep water regime), the effect of water depth on resonant characteristics is too small and can be neglected. While the resonant frequency decreases with the increase of gap width, the amount of this reduction is much more significant in the transitional water regime than that in deep water regime.

- The present study suggests that when evaluating the gap resonance in different draft and gap width, the effect of water depth should be taken into account. The resulting simulations show that the ratio of water depth to body draft is also a governing parameter such that for water depths equal or greater than six times the body draft, the resonant frequency will no longer be influenced by the water depth. Also it is found that the non-dimensional wave heights do not depend on water depth when the latter is more than 10 times the body draft.

\section{AKNOWLEDGEMENTS}

The authors sincerely acknowledge the financial support from Australian Research Council through ARC Discovery Projects Program Grants No. DP110105171 and DP130104535. The first author would like to thank the University of Western Australia for providing the Robert and Maude Gledden scholarship for his $\mathrm{PhD}$ studies and during the course of this study. Financial support from This work was also supported by resources provided by the Pawsey Supercomputing Centre with funding from the Australian Government and the Government of Western Australia. 


\section{REFERENCES}

[1] Miao, G. P., Ishida, H., Saitoh, T., 2000. Influence of gaps between multiple floating bodies on wave forces. China Ocean Eng, 14: 407-422.

[2] Miao, G. P., Saitoh, T., Ishida, H., 2001. Water wave interaction of twin large scale caissons with a small gap between. Coast Eng J, 43:39-58.

[3] Li, B.N., Cheng, L., Deeks, A.J., Teng, B., 2005. A modified scaled boundary finite element method for problems with parallel side-faces. Part II. Application and evaluation. Applied Ocean Research, 27: 224-234.

[4] Zhu, H. R., Zhu, R. C., Miao, G. P., 2008. A time domain investigation on the hydrodynamic resonance phenomena of 3-D multiple floating structures. J Hydrodyn, 20: 611-616.

[5] Sun, L., Eatock Taylor, R., Taylor, P. H., 2010. First and second-order analysis of resonant waves between adjacent barges. J Fluids Struct, 26:954-978.

[6] Buchner, B., Dijk, A., Wilde, J. Numerical multiple body simulations of side-by-side mooring to an FPSO. Proceedings of the 11th international offshore and polar engineering conference, June 17-22, 2001, Stavanger, Norway, ISOPE, vol. 1, pp.343353.

[7] Newman, J. N. Progress in wave load computations on offshore structures. Proceedings of the 23th Conference on Offshore Mechanics and Arctic Engineering. New York: ASME, 2004.

[8] Chen, X. B. Hydrodynamic analysis for offshore LNG terminals. Second International workshop on applied offshore Hydrodynamics. Rio De Janeiro, Brazil, 14-15, April, 2005. 
[9] Molin, B., Remy, F., Camhi, A., Ledoux, A. Experimental and numerical study of the gap resonances in-between two rectangular barges. 13th congress of International Maritime Association of Mediterranean IMAM 2009, October 12-15, 2009 Istanbul, Turkey, pp.689-696.

[10] Pauw, W. H., Huijsmans, R. H., \& Voogt, A. Advances in the hydrodynamics of sideby-side moored vessels. Proceedings of the 26th International Conference on Offshore Mechanics and Arctic Engineering. 2007, San Diego, USA.

[11] Bunnik, T., Pauw, W., Voogt, A. Hydrodynamic analysis for side-by-side offloading. Proceedings of the 19th International Offshore and Polar Engineering Conference, Osaka, Japan. June 21-26, 2009.

[12] Lu, L., Teng, B., Cheng, L., Sun, L., Chen, X., 2011. Modeling of multi bodies in close proximity under water waves- Fluid resonance in narrow gaps. Science China, 54: 16-25.

[13] Chen, X. B., Duan, WY., Liu, HX. Dissipation effect in potential flows of fairly perfect fluid. Proceedings of 26th international workshop on water waves and floating bodies, April 17-20, 2011, Athens, Greece.

[14] Lu, L., Cheng, L., Teng B., Li, Y. Numerical simulation of hydrodynamic resonance in a narrow gap between twin bodies subject to water waves. Proceedings of the 18th International Offshore and Polar Engineering Conference. Cupertino: ISOPE, 2008, 114119.

[15] Lu, L., Cheng, L., Teng, B., Zhao, M., 2010a. Numerical investigation of fluid resonance in two narrow gaps of three identical rectangular structures. Applied Ocean Researsh, 32: $177-190$. 
[16] Lu, L., Cheng, L., Teng, B., Sun, L., 2010b. Numerical simulation and comparison of potential flow and viscous fluid models in near trapping of narrow gaps. Journal of Hydrodynamics, 22(5):120-125.

[17] Sauder, T., Kristiansen, T., Ostman, A. Validation of a numerical method for the study of piston like oscillations between a ship and a terminal. In: Proceedings of twentieth International offshore and polar engineering conference, Beijing, China, 2010.

[18] Moradi, N., Zhou, T., Cheng, L., 2015. Effect of inlet configuration on wave resonance in the narrow gap of two fixed bodies in close proximity. Ocean Engineering, 103: 88102.

[19] Saitoh, T., Miao, G. P., Ishida, H. Theoretical analysis on appearance condition of fluid resonance in a narrow gap between two modules of very large floating structure. Proceedings of the 3rd Asia-Pacific Workshop on Marine Hydrodynamics. Beijing China, 2006.

[20] Liu, Y., Li, H., 2013. A new semi-analytical solution for gap resonance between twin rectangular boxes. J Engineering for Maritime Environment, 0 (0), 1-14.

[21] Kristiansen, T., Faltinsen, O.M. Resonant water motion between a ship and a terminal in shallow water. Proceedings of 26th International Conference on Offshore Mechanics and Arctic Engineering, 2007, San Diego, USA.

[22] Jacobsen, N.G., Fuhrman, D.R., Fredsøe, J. A., 2011. Wave generation toolbox for the open-source CFD library: OpenFoam. Int. J. Numer. Meth. Fluids, 70 (9) 1073-1088.

[23] HydroSTAR For Experts. http://www.veristar.com

[24] Hirt, C.W., Nichols, B.D., 1981. Volume of fluid (VOF) method for the dynamics of free boundaries. Journal of Computational Physics, 39(1):201-225.

[25] Berberović, E., Van Hinsberg, N.P., Jakirlić, S., Roisman, I.V., Tropea, C., 2009. Drop 
impact onto a liquid layer of finite thickness: dynamics of the cavity evolution. Physical Review E-Statistical, Nonlinear, and Soft Matter Physics, 79(3):1-15. Art. No: 036 360.

Table 1. Different setup parameters for the study of coupled effect of draft and water depth

\begin{tabular}{|c|c|c|c|c|}
\hline Case No. & $\boldsymbol{B g}(\boldsymbol{m})$ & $\boldsymbol{d}$ & $\boldsymbol{h}(\boldsymbol{m})$ & $\boldsymbol{B}(\boldsymbol{m})$ \\
\hline Case 1 & 0.05 & 0.15 & 0.5 & 0.5 \\
\hline Case 2 & 0.05 & 0.15 & 0.75 & 0.5 \\
\hline Case 3 & 0.05 & 0.15 & 1 & 0.5 \\
\hline Case 4 & 0.05 & 0.15 & 1.5 & 0.5 \\
\hline Case 5 & 0.05 & 0.15 & 2 & 0.5 \\
\hline Case 6 & 0.05 & 0.25 & 0.5 & 0.5 \\
\hline Case 7 & 0.05 & 0.25 & 0.75 & 0.5 \\
\hline Case 8 & 0.05 & 0.25 & 1 & 0.5 \\
\hline Case 9 & 0.05 & 0.25 & 1.5 & 0.5 \\
\hline Case 10 & 0.05 & 0.25 & 2 & 0.5 \\
\hline Case 11 & 0.05 & 0.35 & 0.5 & 0.5 \\
\hline Case 12 & 0.05 & 0.35 & 0.75 & 0.5 \\
\hline Case 13 & 0.05 & 0.35 & 1 & 0.5 \\
\hline Case 14 & 0.05 & 0.35 & 1.5 & 0.5 \\
\hline Case 15 & 0.05 & 0.35 & 2 & 0.5 \\
\hline
\end{tabular}

Table 2. Different setup parameters for the study of coupled effect of gap width and water depth

\begin{tabular}{|c|c|c|c|c|}
\hline Case No. & $\boldsymbol{B g}(\boldsymbol{m})$ & $\boldsymbol{d}$ & $\boldsymbol{h}(\boldsymbol{m})$ & $\boldsymbol{B}(\boldsymbol{m})$ \\
\hline Case 1 & 0.025 & 0.25 & 0.5 & 0.5 \\
\hline Case 2 & 0.025 & 0.25 & 0.75 & 0.5 \\
\hline Case 3 & 0.025 & 0.25 & 1 & 0.5 \\
\hline Case 4 & 0.025 & 0.25 & 1.5 & 0.5 \\
\hline Case 5 & 0.025 & 0.25 & 2 & 0.5 \\
\hline Case 6 & 0.075 & 0.25 & 0.5 & 0.5 \\
\hline Case 7 & 0.075 & 0.25 & 0.75 & 0.5 \\
\hline Case 8 & 0.075 & 0.25 & 1 & 0.5 \\
\hline Case 9 & 0.075 & 0.25 & 1.5 & 0.5 \\
\hline Case 10 & 0.075 & 0.25 & 2 & 0.5 \\
\hline Case 11 & 0.1 & 0.25 & 0.5 & 0.5 \\
\hline Case 12 & 0.1 & 0.25 & 0.75 & 0.5 \\
\hline Case 13 & 0.1 & 0.25 & 1 & 0.5 \\
\hline Case 14 & 0.1 & 0.25 & 1.5 & 0.5 \\
\hline Case 15 & 0.1 & 0.25 & 2 & 0.5 \\
\hline
\end{tabular}




\section{Figures}
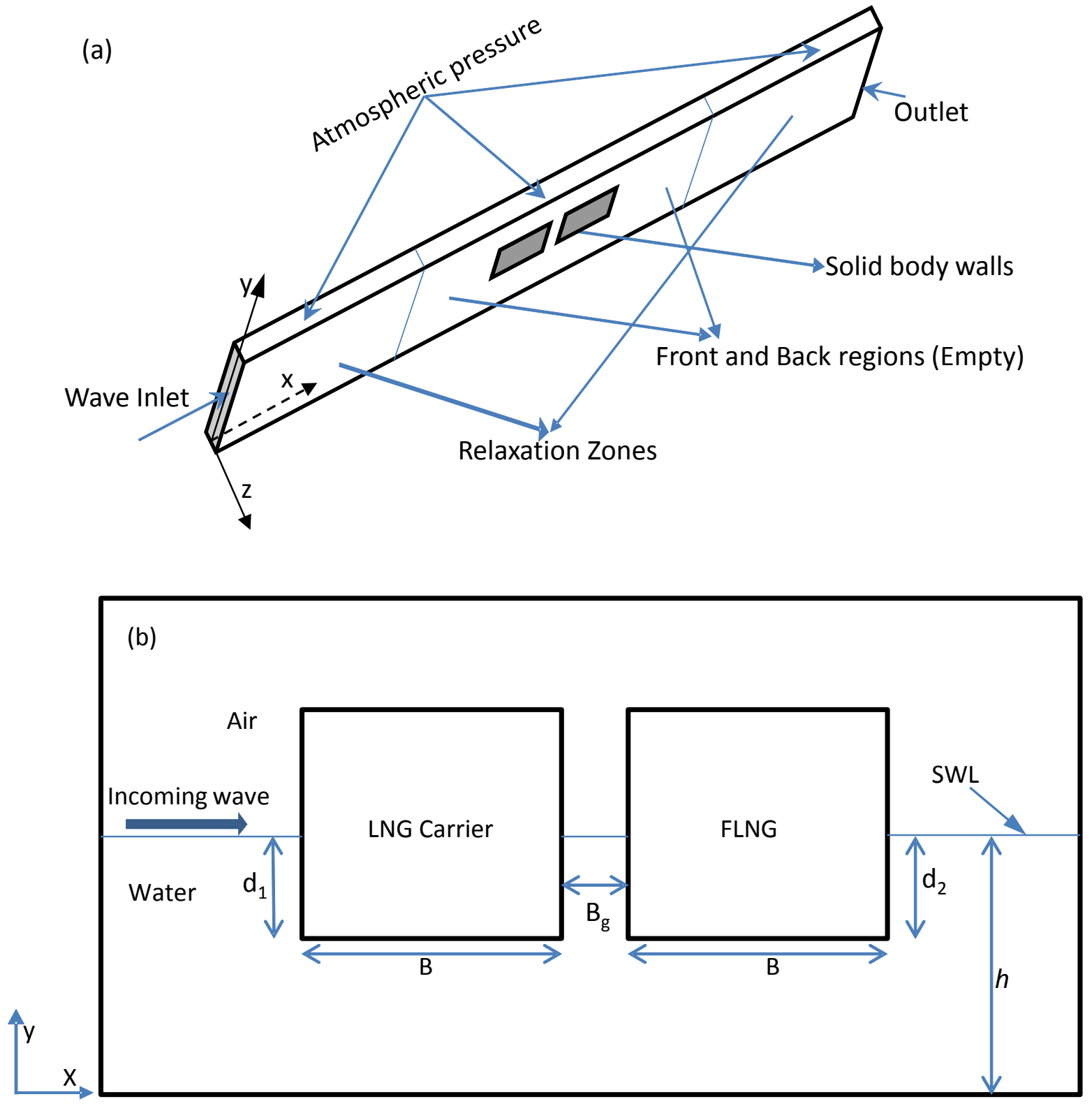

Figure 1. (a) Simulation domain and the definition of the co-ordinate system; (b) Sketch of the floating bodies and the definition of the geometric parameters. 


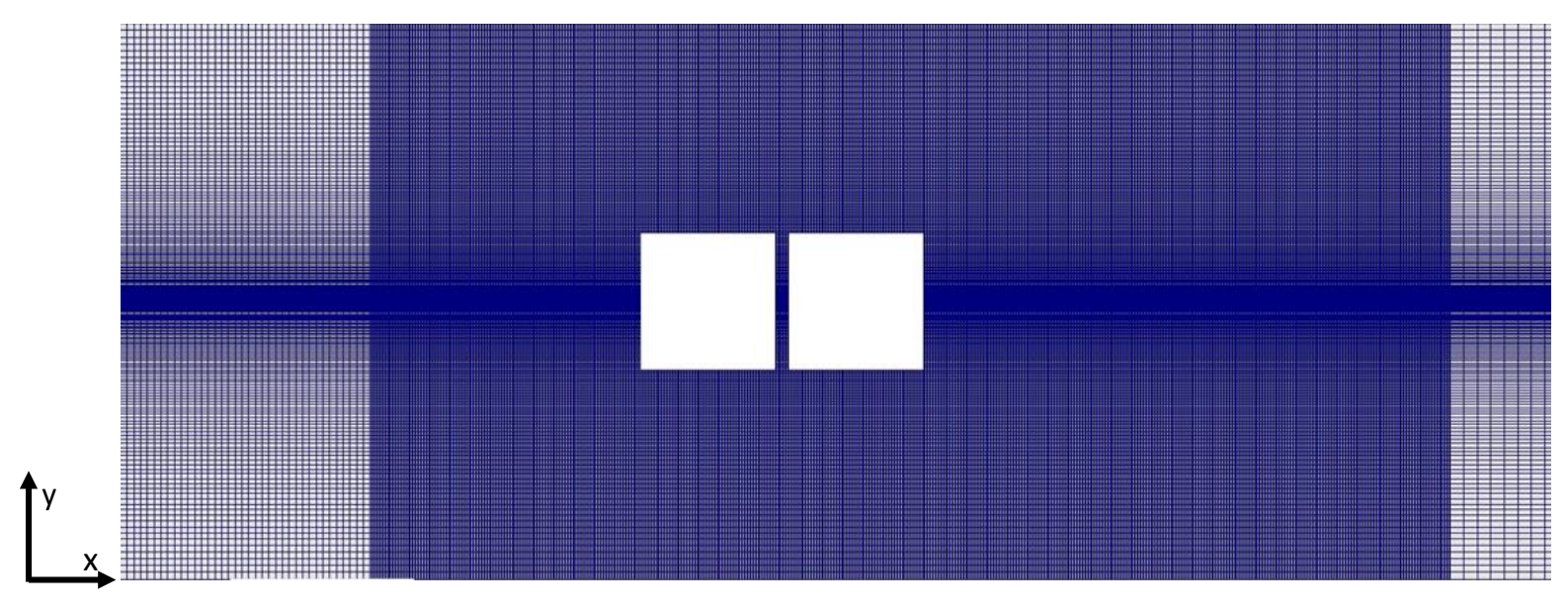

Figure 2. Side view of the sample meshes around the bodies. 


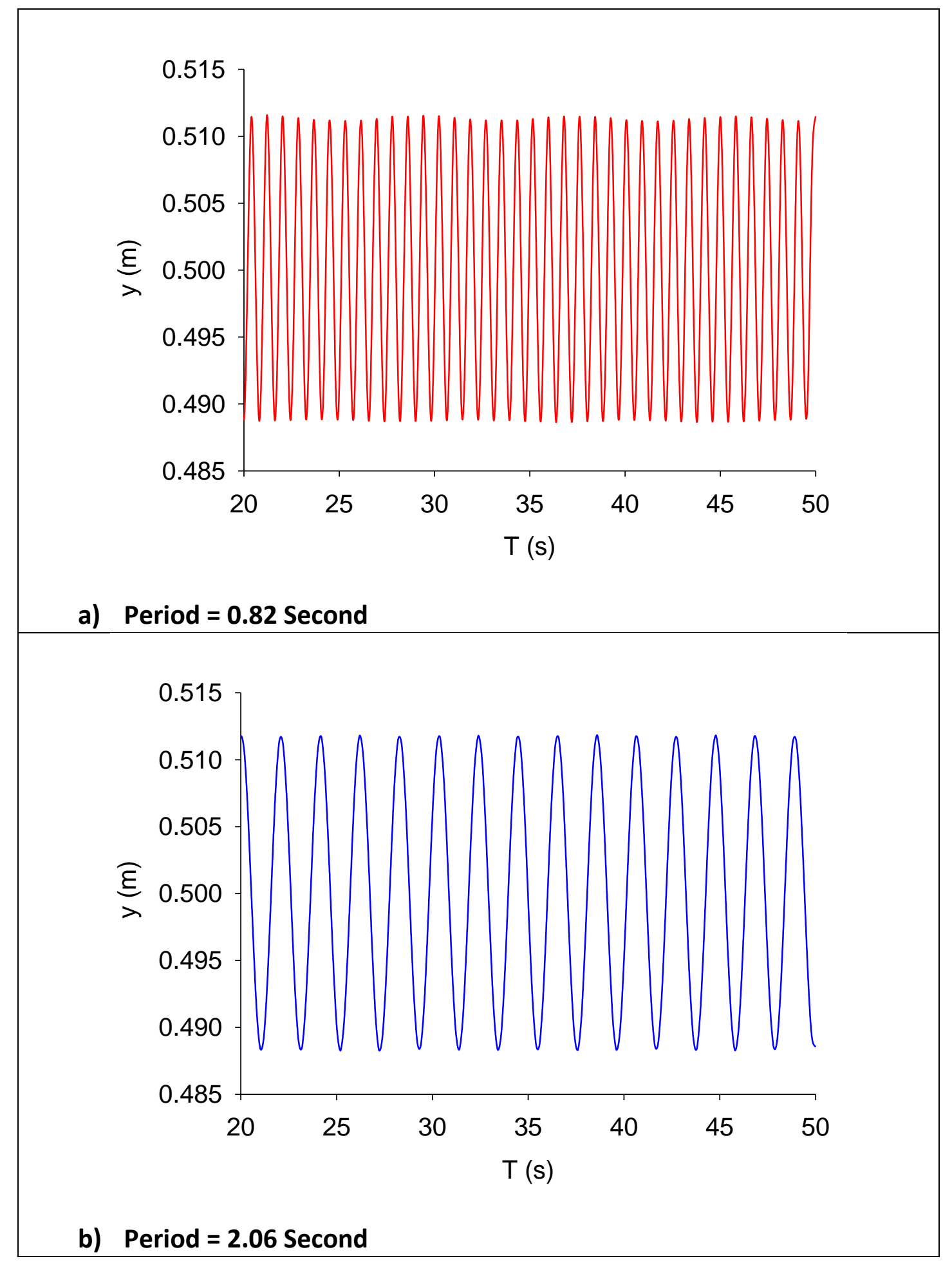

Figure 3. Time series of the water elevation for the shortest and longest waves at $x=6 \mathrm{~m}$. (a) Period $=0.82$ Second; (b) Period $=2.06$ Seconds. 


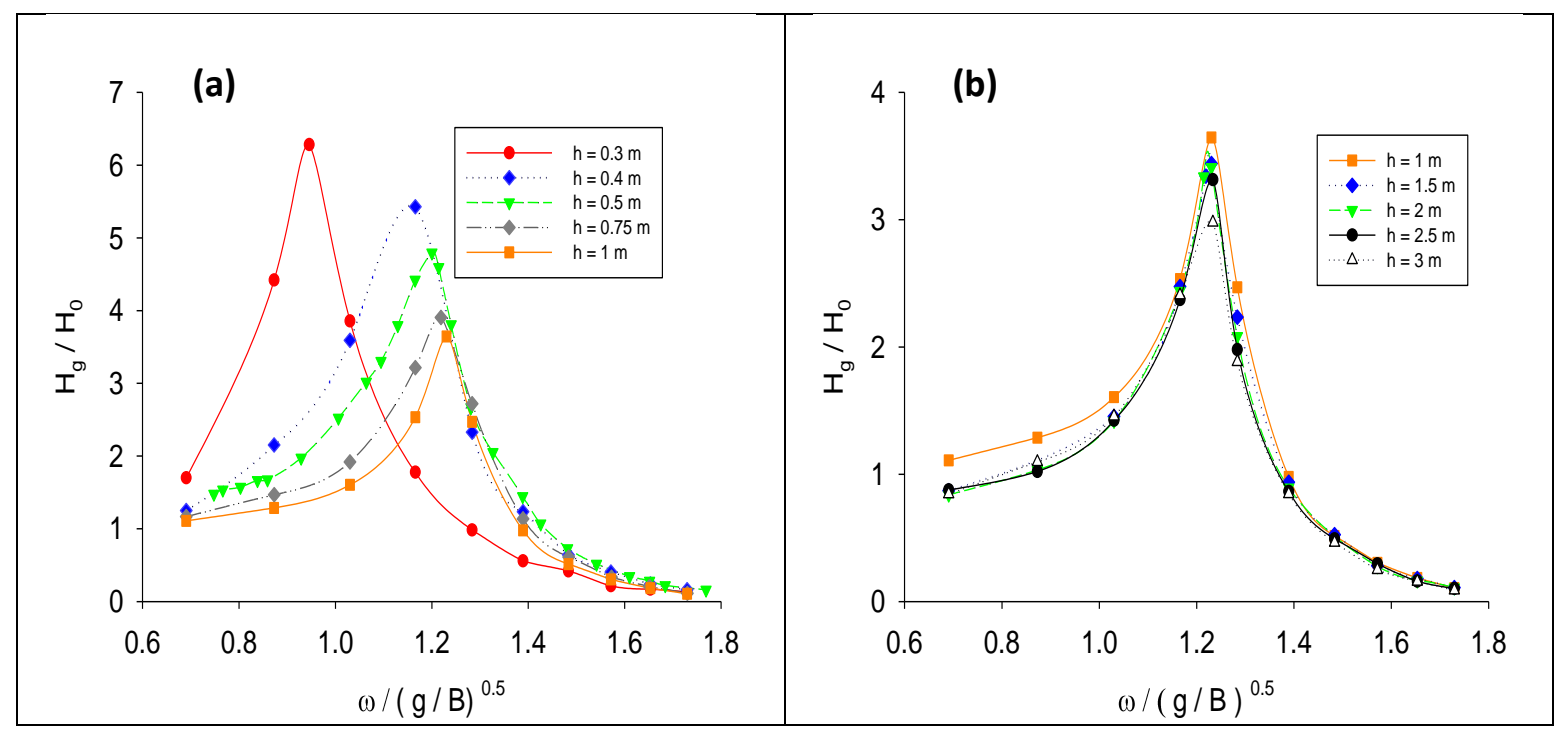

Figure 4. Non-dimensional wave height as a function of wave frequency for different water depth $h$ and

for $d=0.25 \mathrm{~m}$ and $B_{g}=0.05 \mathrm{~m}$. (a) Shallow and transitional water regimes; (b) deep water regime.

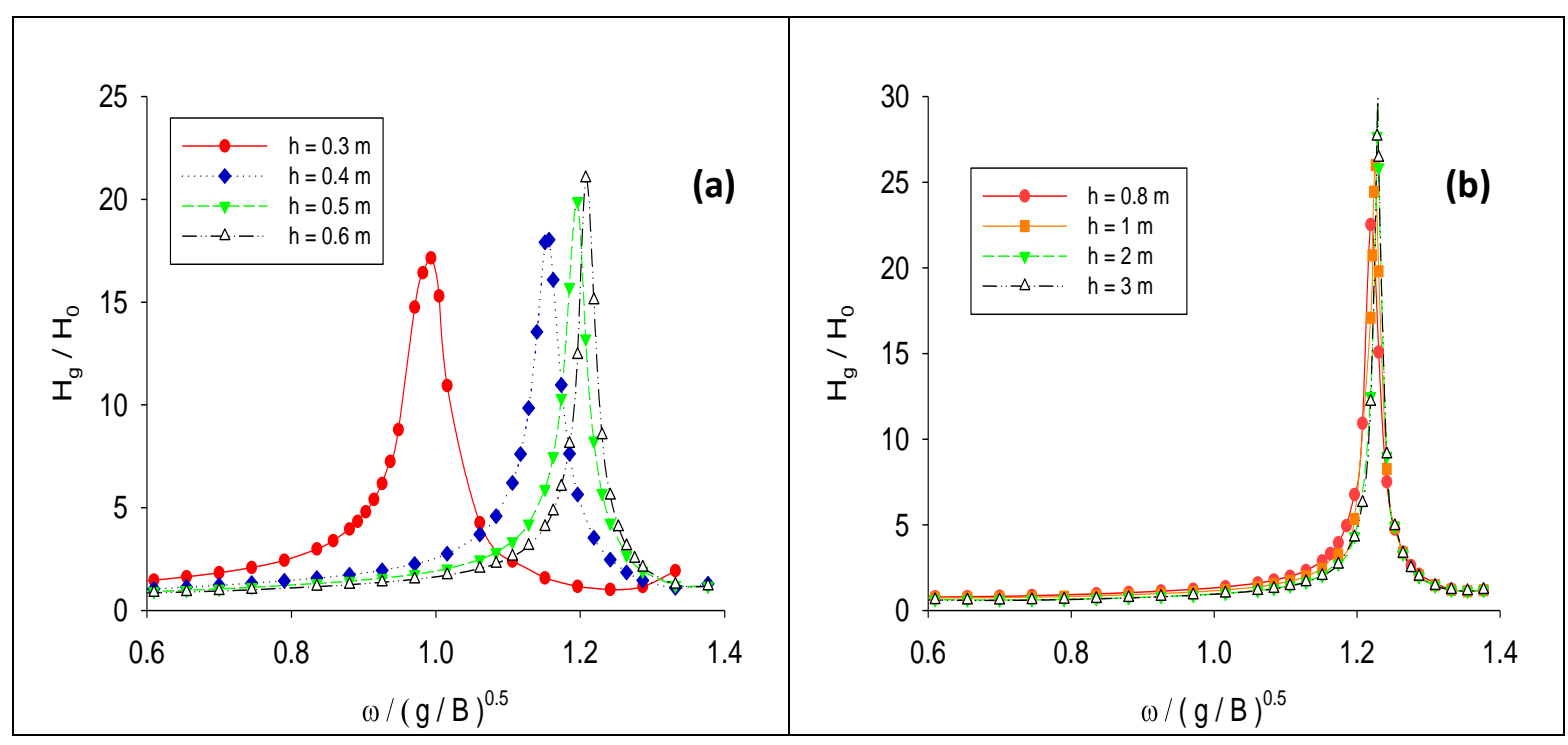

Figure 5. Non-dimensional wave height as a function of wave frequency for different water depth $h$ predicted using the Potential flow model for $d=0.25 \mathrm{~m}$ and $B_{g}=0.05 \mathrm{~m}$. (a) Shallow and transitional water regime; (b) Transitional and deep water regimes. 


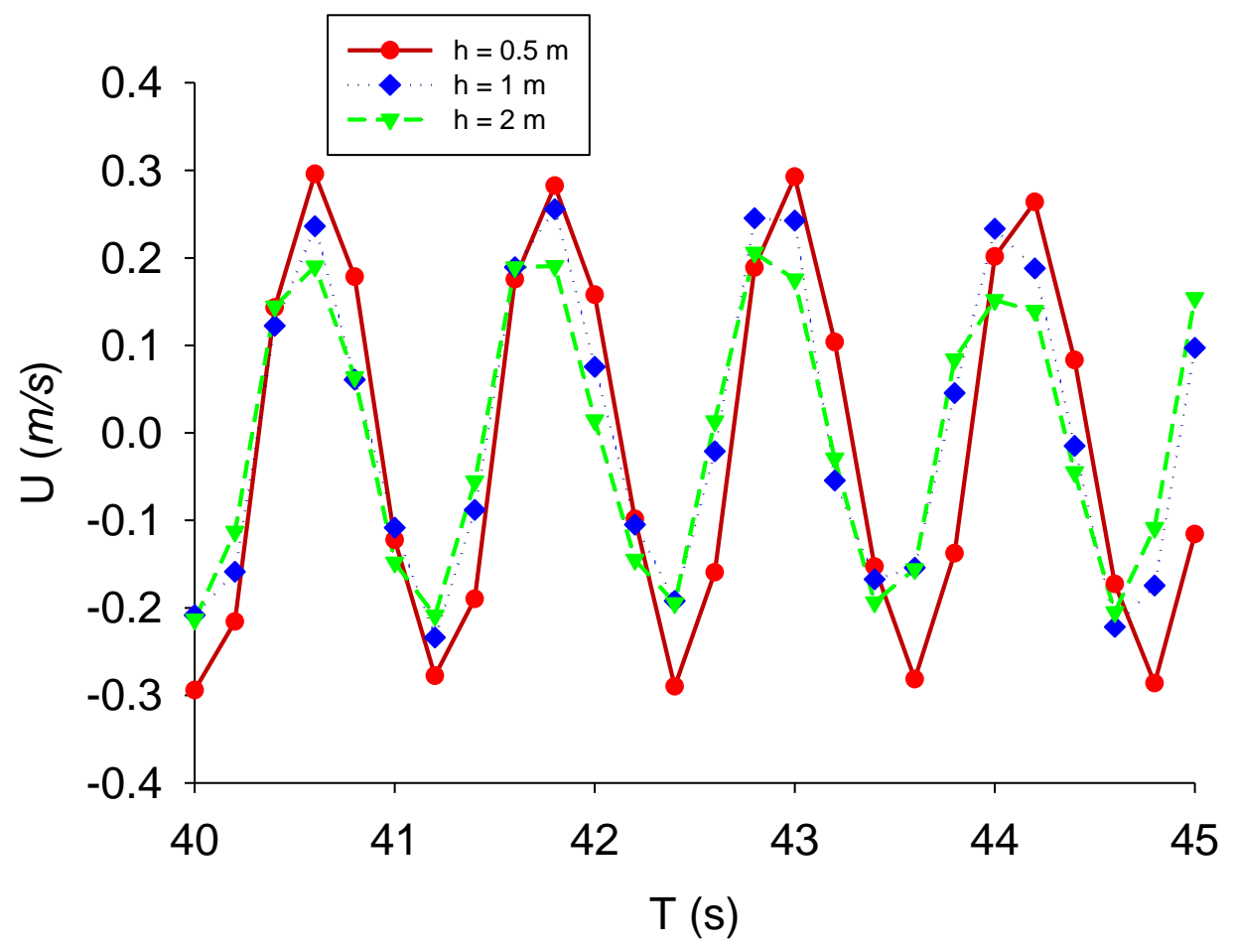

Figure 6. Dependence on water depth of the surface velocity in the middle of the gap, for $d=0.25 \mathrm{~m}$ and $B_{g}=0.05 \mathrm{~m}$.

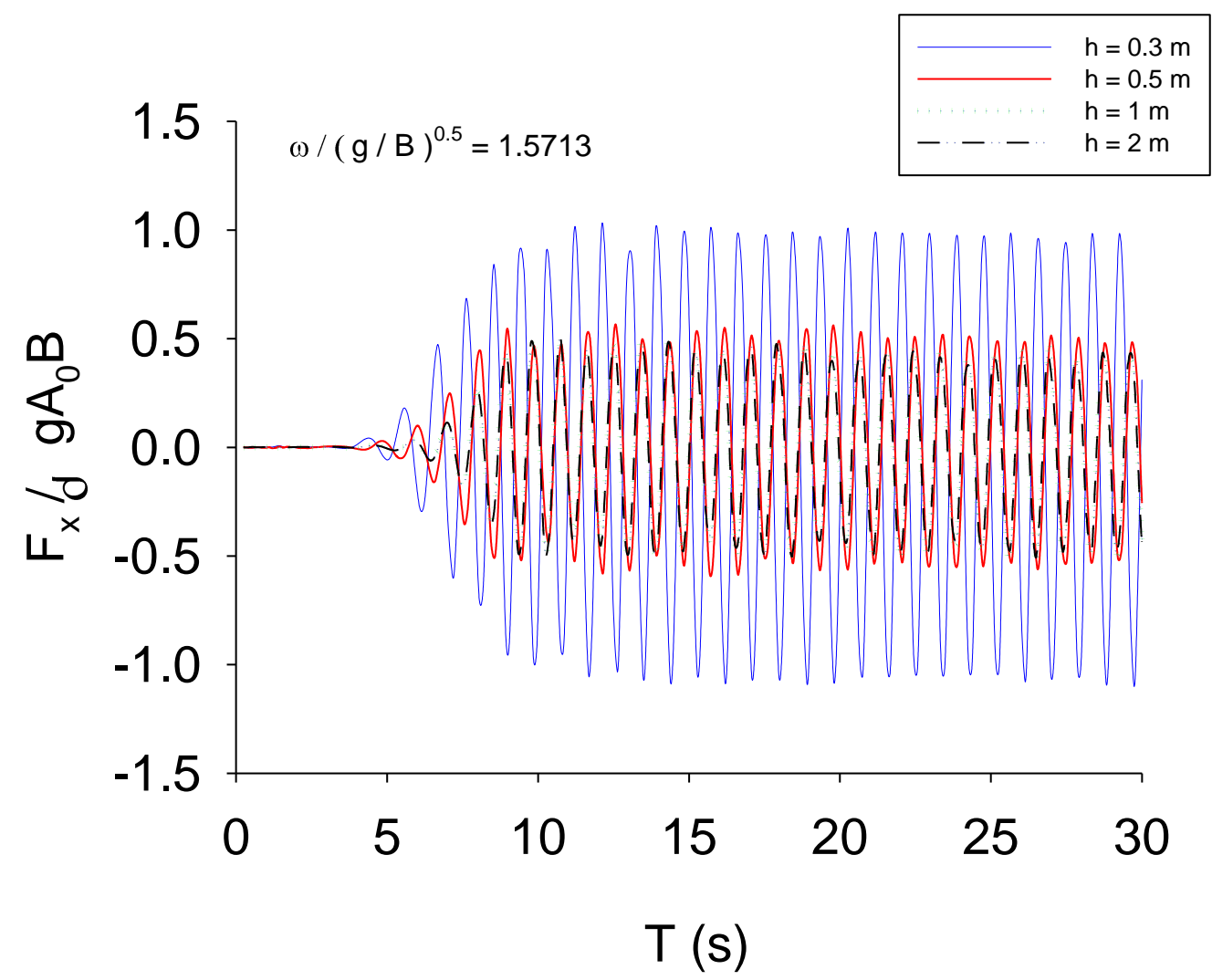


Figure 7. Time series of the horizontal force on the front body at different water depths for $d=$ $0.25 \mathrm{~m}$ and $B_{g}=0.05 \mathrm{~m}$.

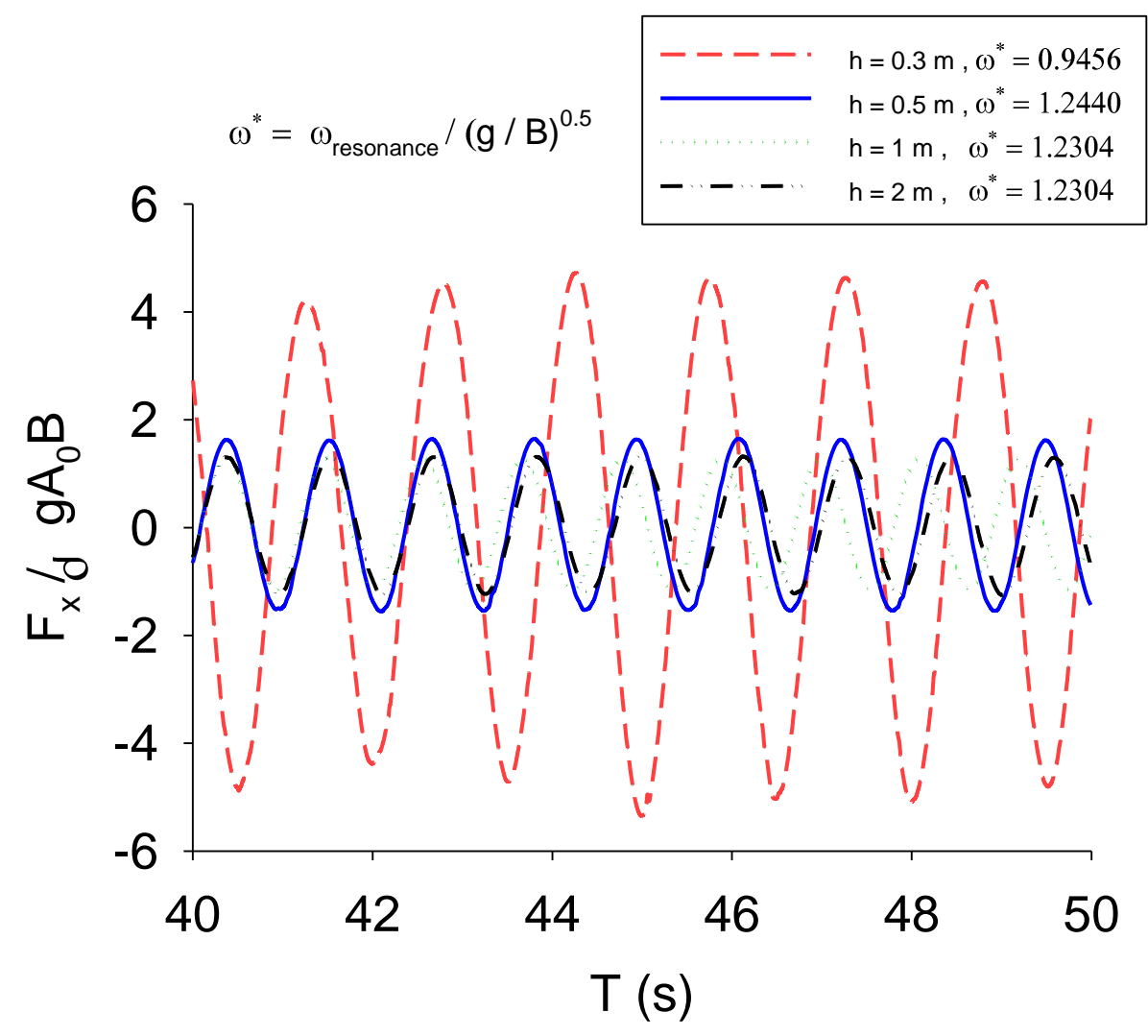

Figure 8. Time series of the horizontal force on the front body at resonant frequency of different water depths for $d=0.25 \mathrm{~m}$ and $B_{g}=0.05 \mathrm{~m}$. 


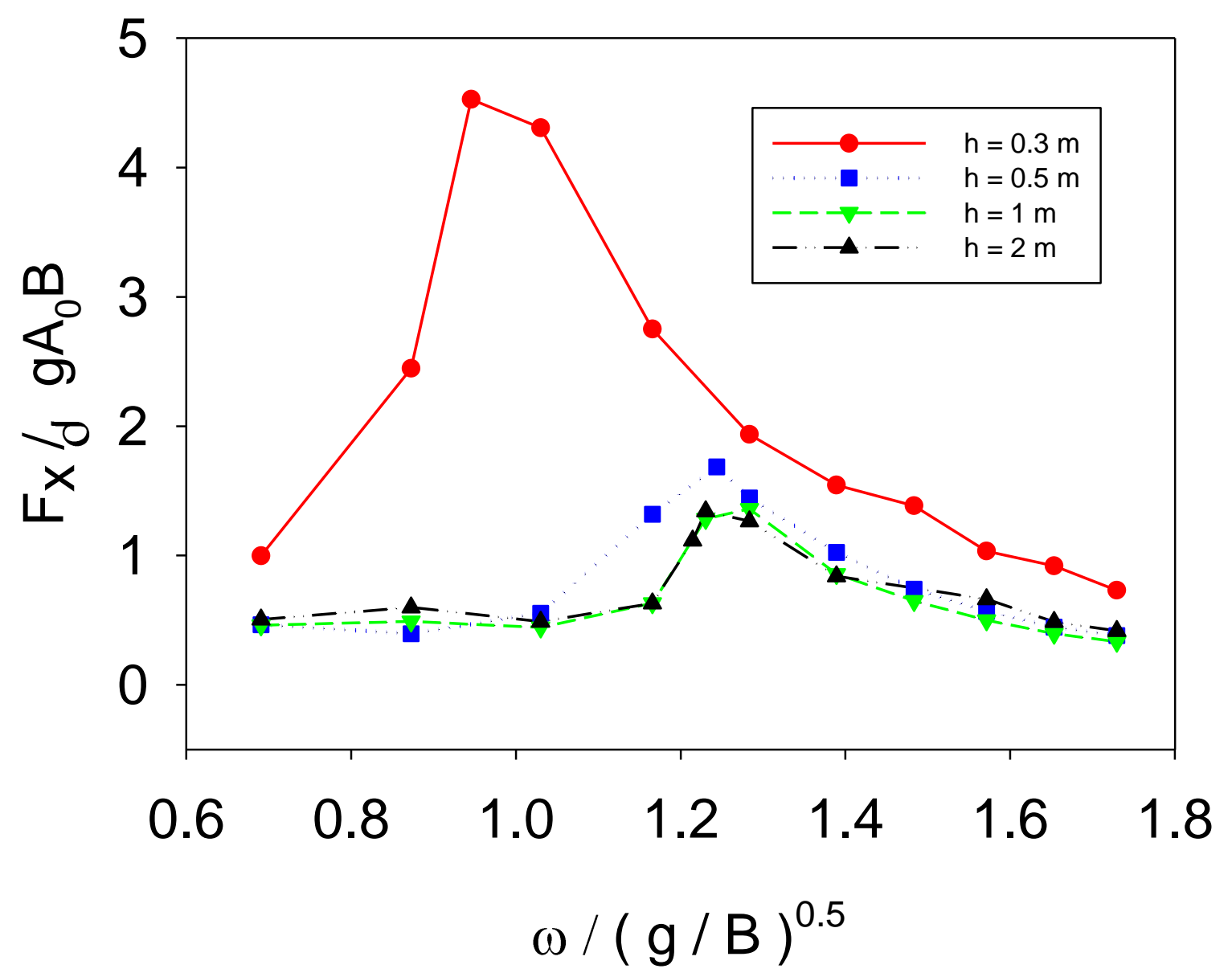

Figure 9. Variation of the maximum magnitude of the non-dimensional horizontal force with respect to the non-dimensional wave frequency at different water depths for $d=0.25 \mathrm{~m}$ and $B_{g}=0.05 \mathrm{~m}$. 


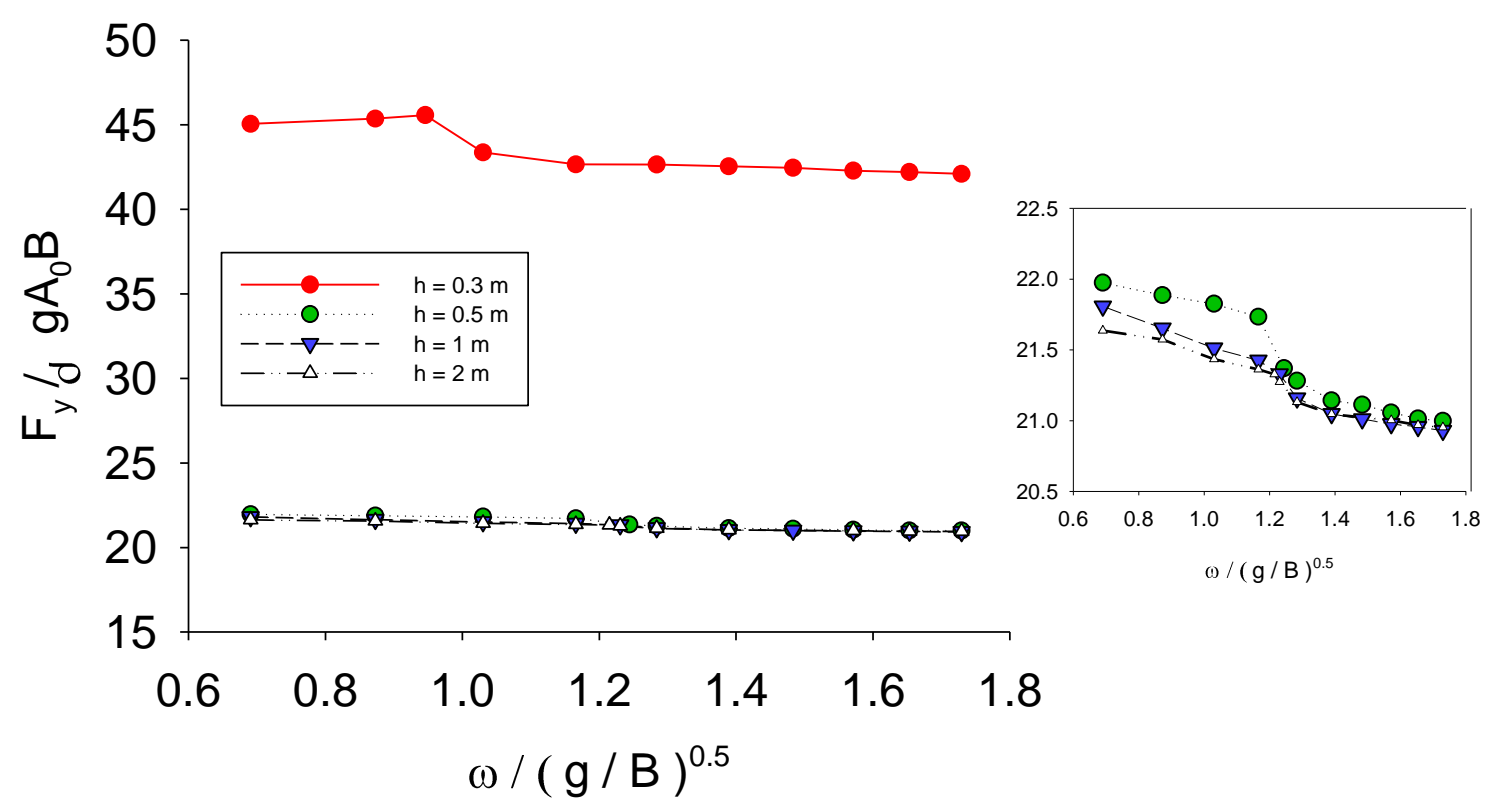

Figure 10. Variation of the maximum magnitude of the non-dimensional vertical force with respect to the non-dimensional wave frequency at different water depths for the typical case with $d=0.25 \mathrm{~m}$ and $B_{g}=0.05 \mathrm{~m}$.

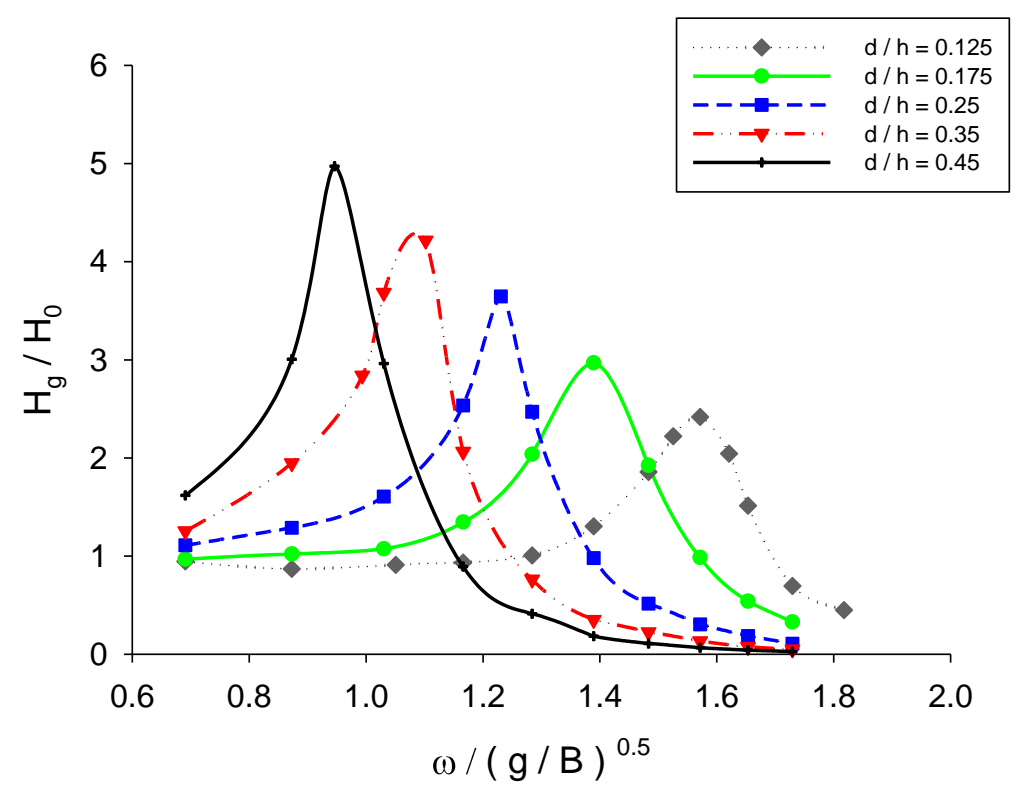

Figure 11. Non-dimensional wave height as a function of wave frequency for different draft to height ratios at $\mathrm{h}=1 \mathrm{~m}$ and $B_{g}=0.05 \mathrm{~m}$. 


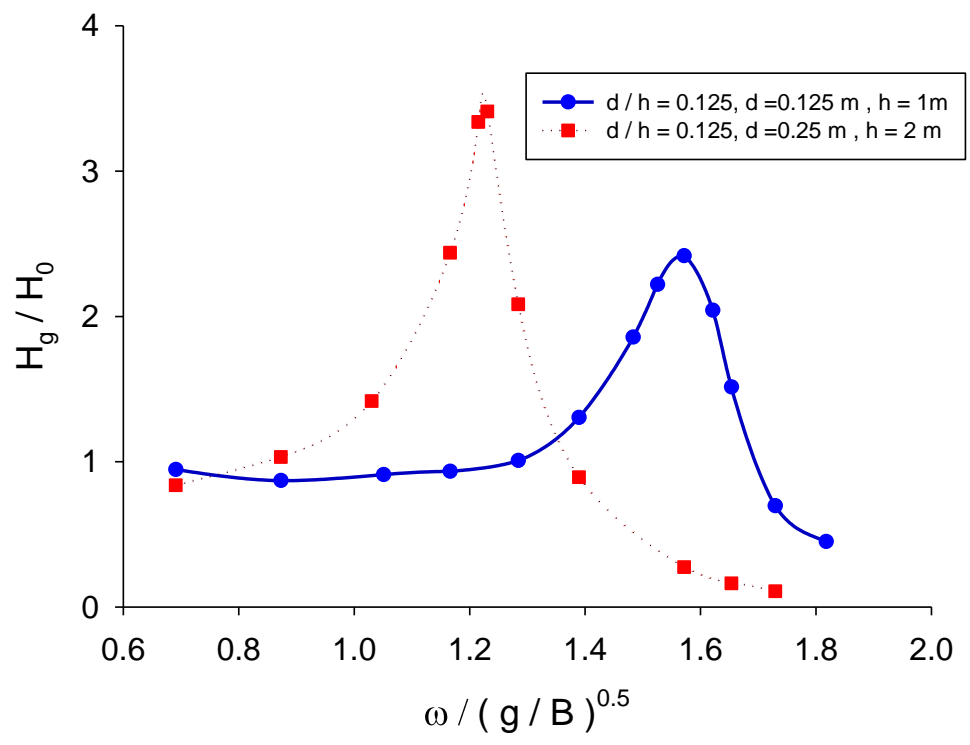

Figure 12. Comparison of the gap wave height obtained at the same draft to water depth ratio but different drafts with $B_{g}=0.05 \mathrm{~m}$.

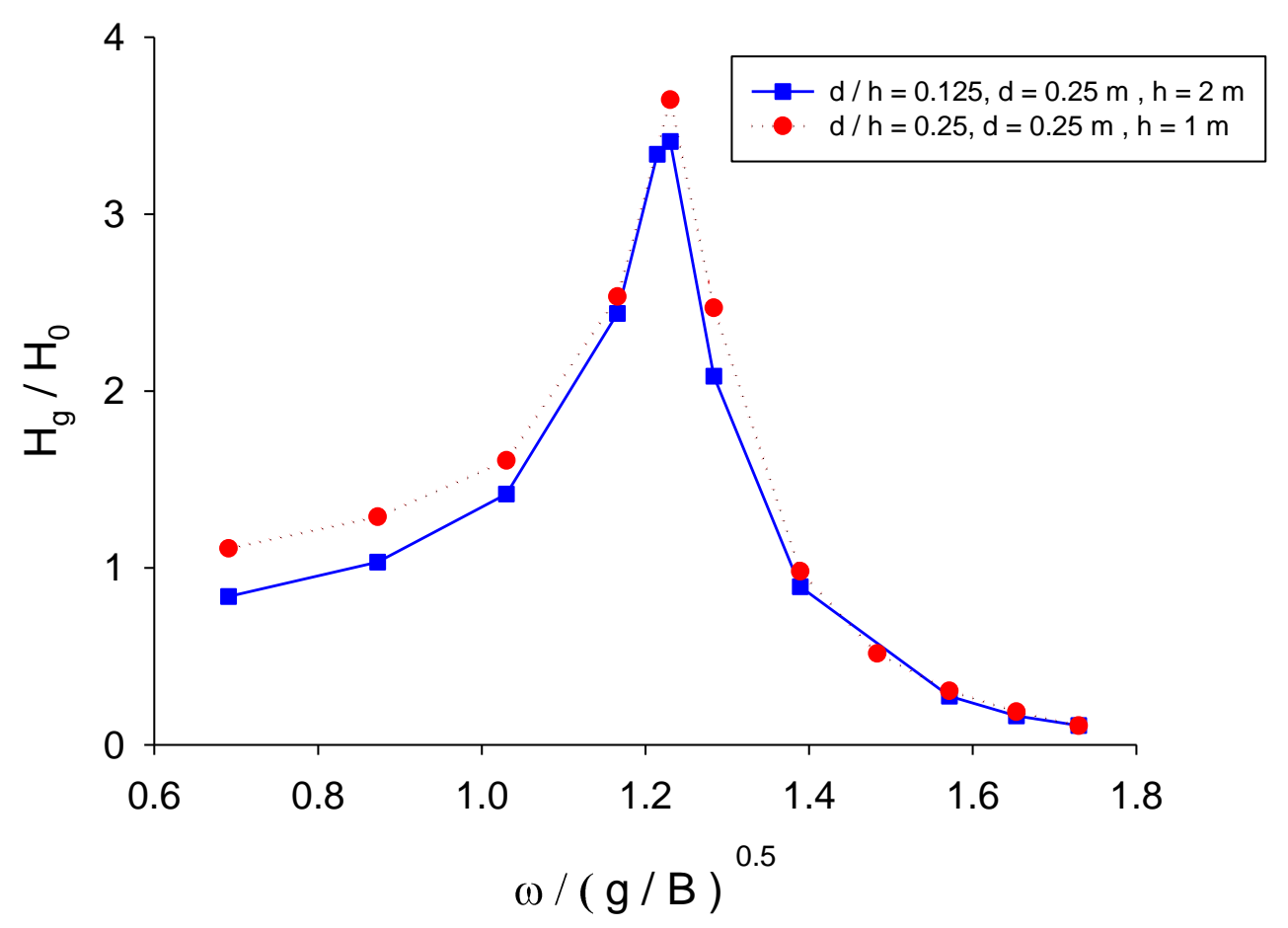

Figure 13. Comparison of the gap wave height obtained at different draft to water depth ratios but the same drafts with $B_{g}=0.05 \mathrm{~m}$. 


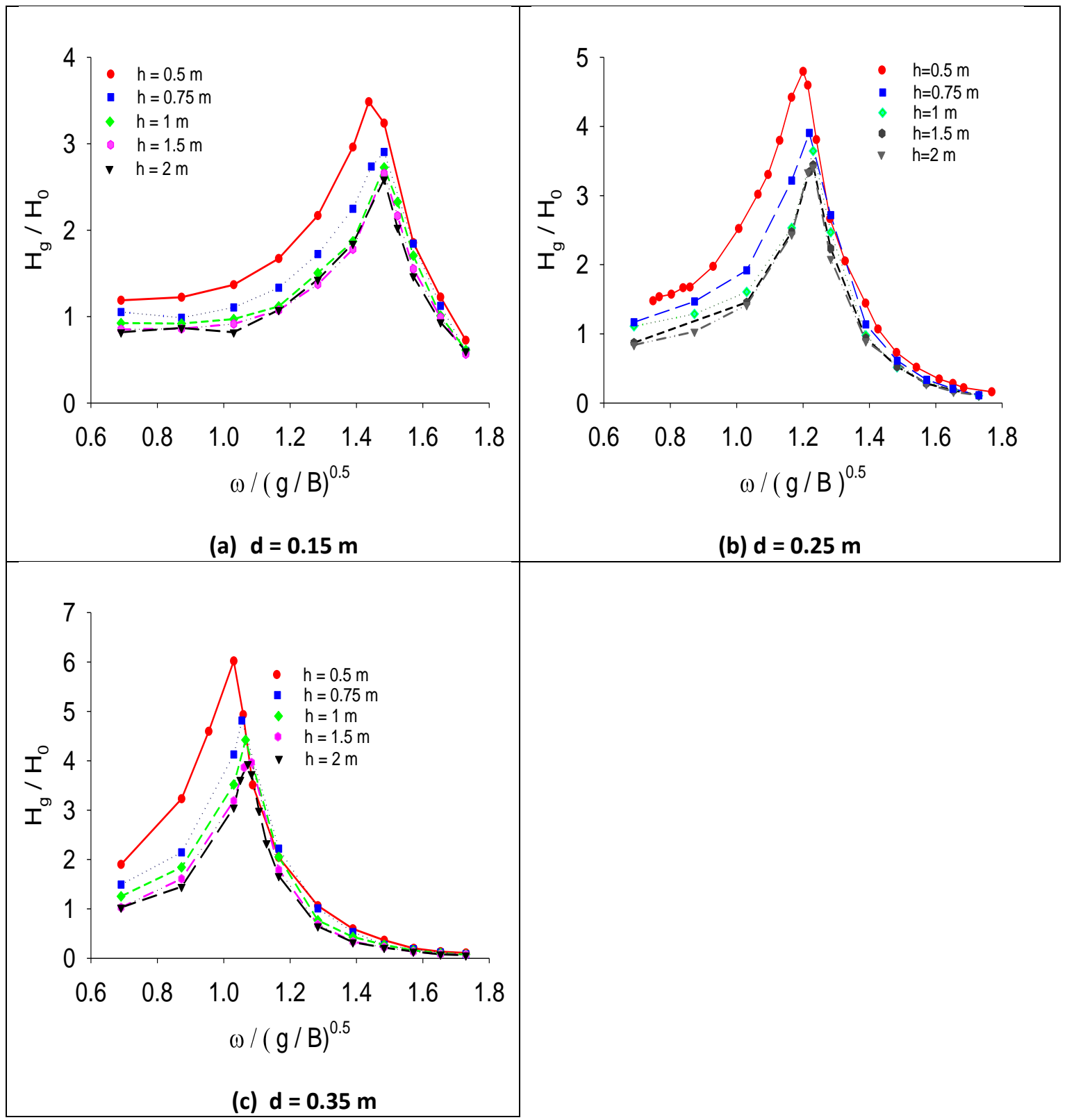

Figure 14. Variation of the non-dimensional wave height as a function of normalized frequency for different water depths and body drafts at $B_{g}=0.05 \mathrm{~m}$. 


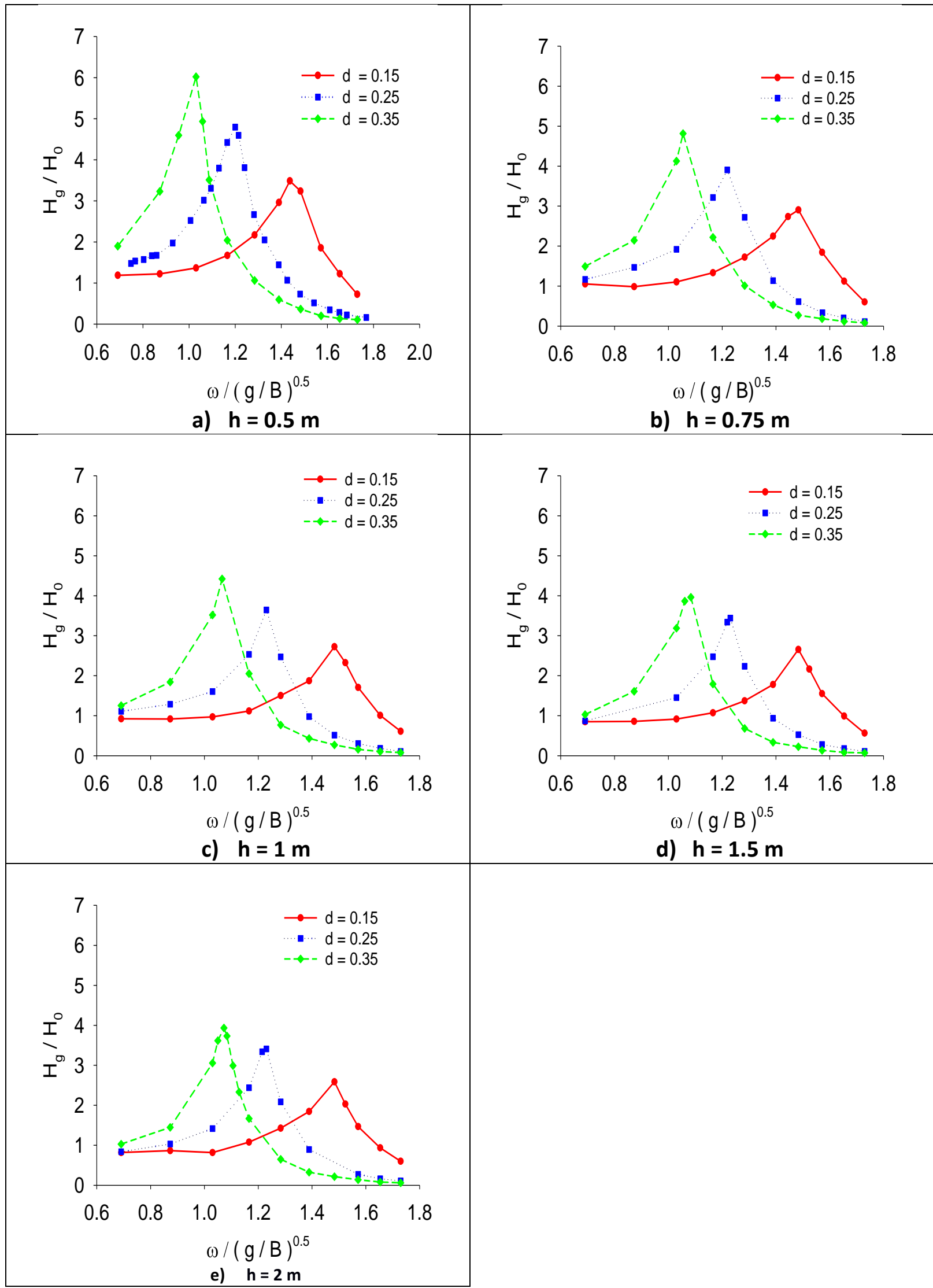

Figure 15. Variation of the non-dimensional wave height as a function of normalized frequency for different body drafts and water depths at $B_{g}=0.05 \mathrm{~m}$. 


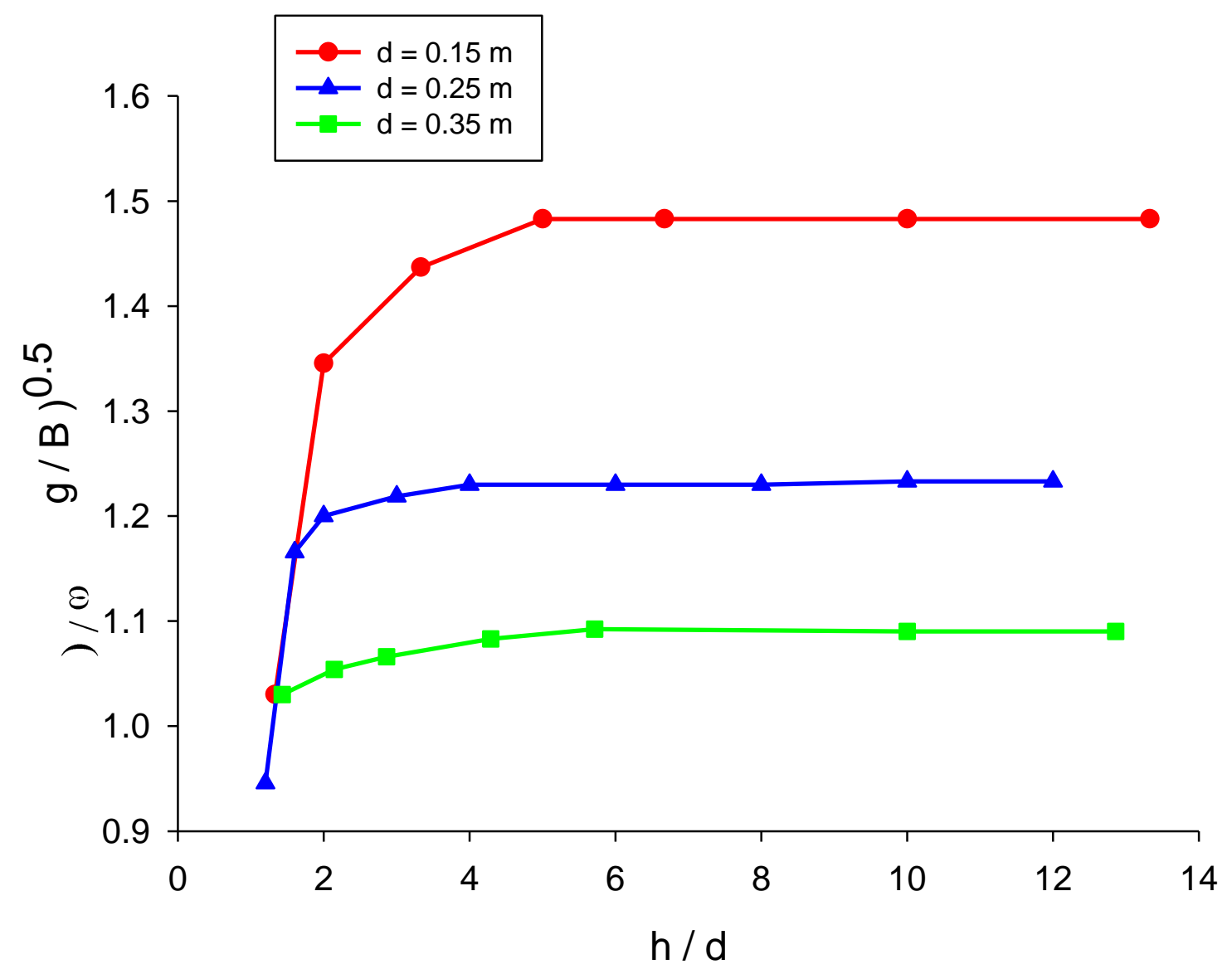

Figure 16. Comparison of resonant frequency as a function of normalized water depth for different body drafts at $B_{g}=0.05 \mathrm{~m}$. 


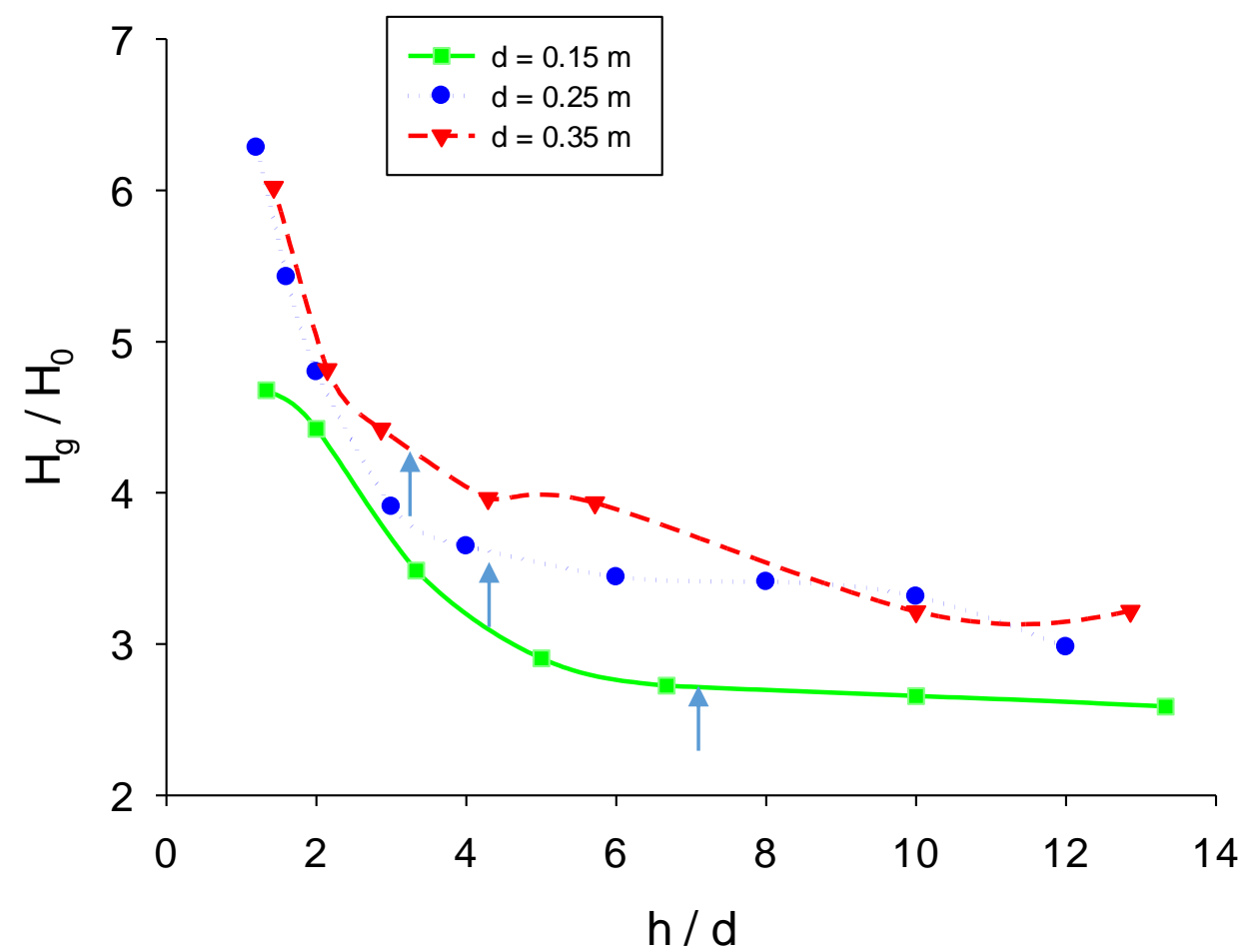

Figure 17. Comparison of resonant wave height as a function of normalized water depth for different body drafts at $B_{g}=0.05 \mathrm{~m}$. The arrows indicate the critical water depth for each body draft.

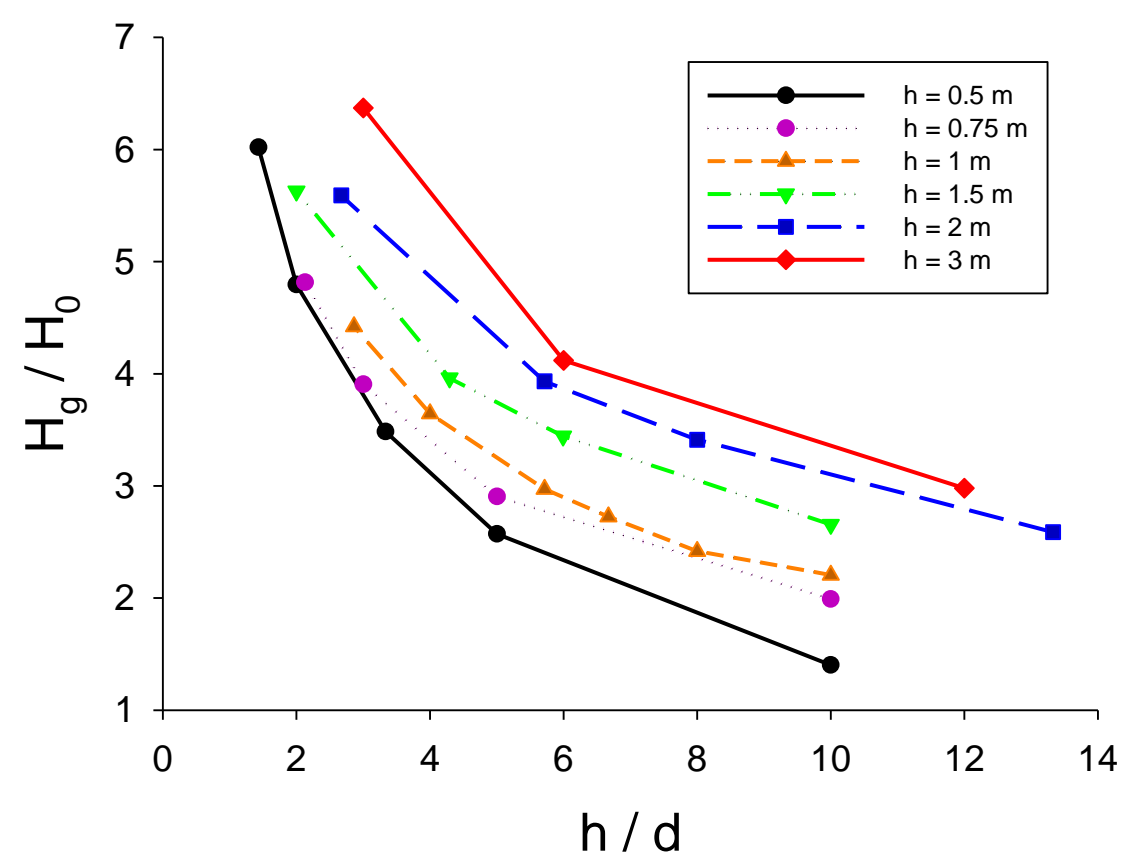

Figure 18. Comparison of resonant wave height as a function of normalized water depth for different water depths at $B_{g}=0.05 \mathrm{~m}$. 


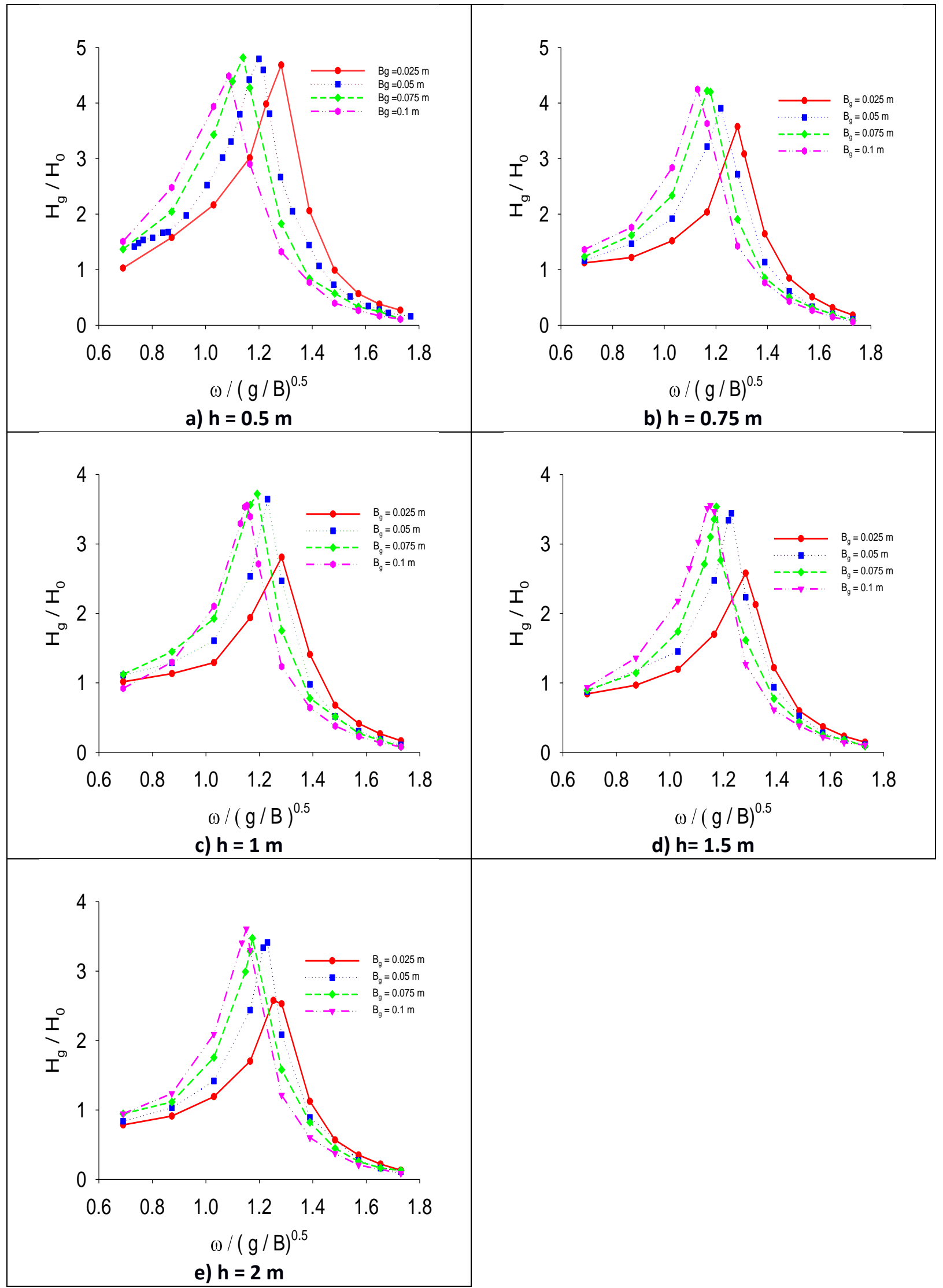

Figure 19. Variation of the non-dimensional wave height as a function of normalized frequency for different gap widths and water depths at $\mathrm{d}=0.25 \mathrm{~m}$. 


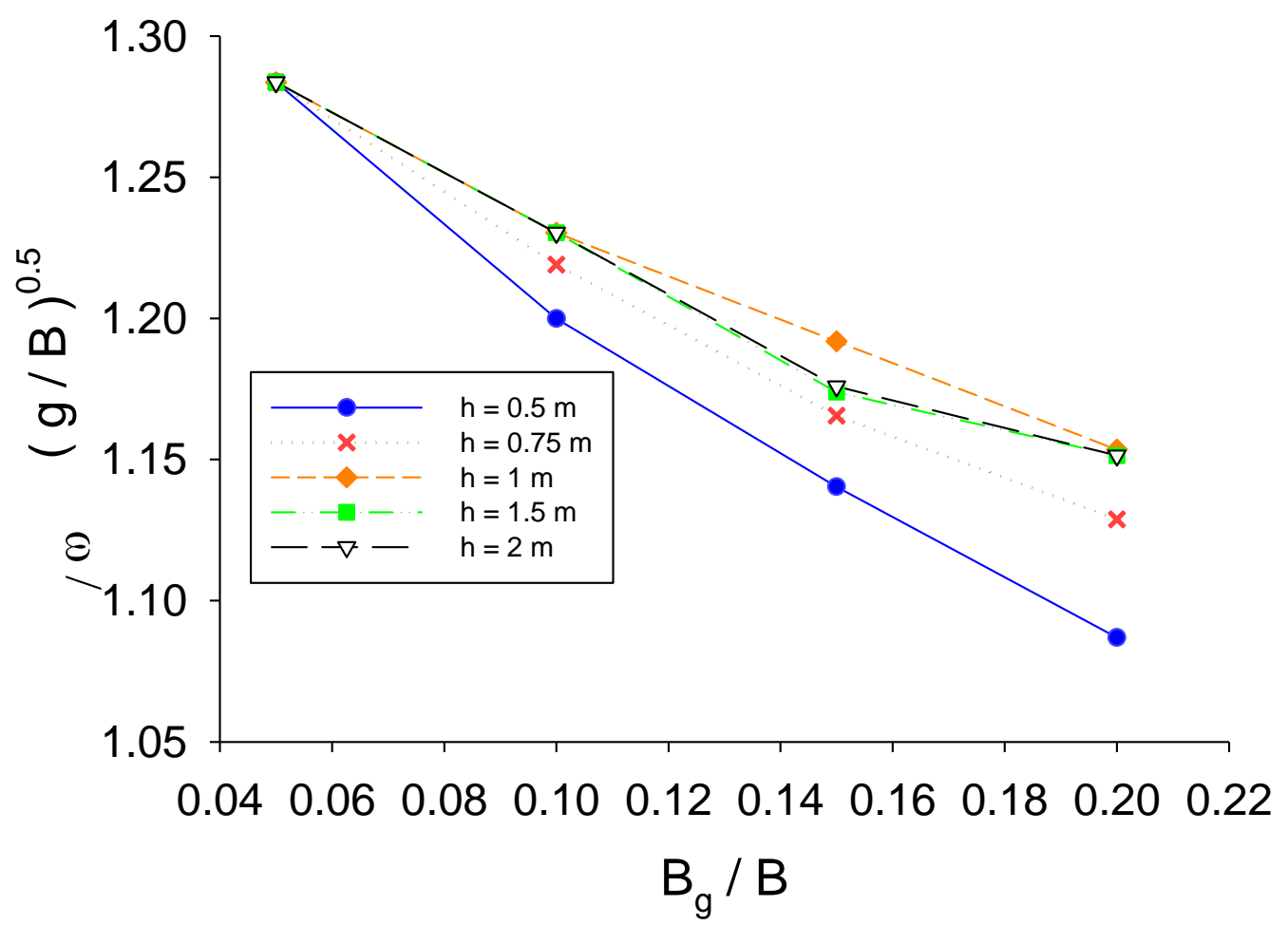

Figure 20. Comparison of resonant frequency as a function of normalized gap width for different water depths at $\mathrm{d}=0.25 \mathrm{~m}$. 


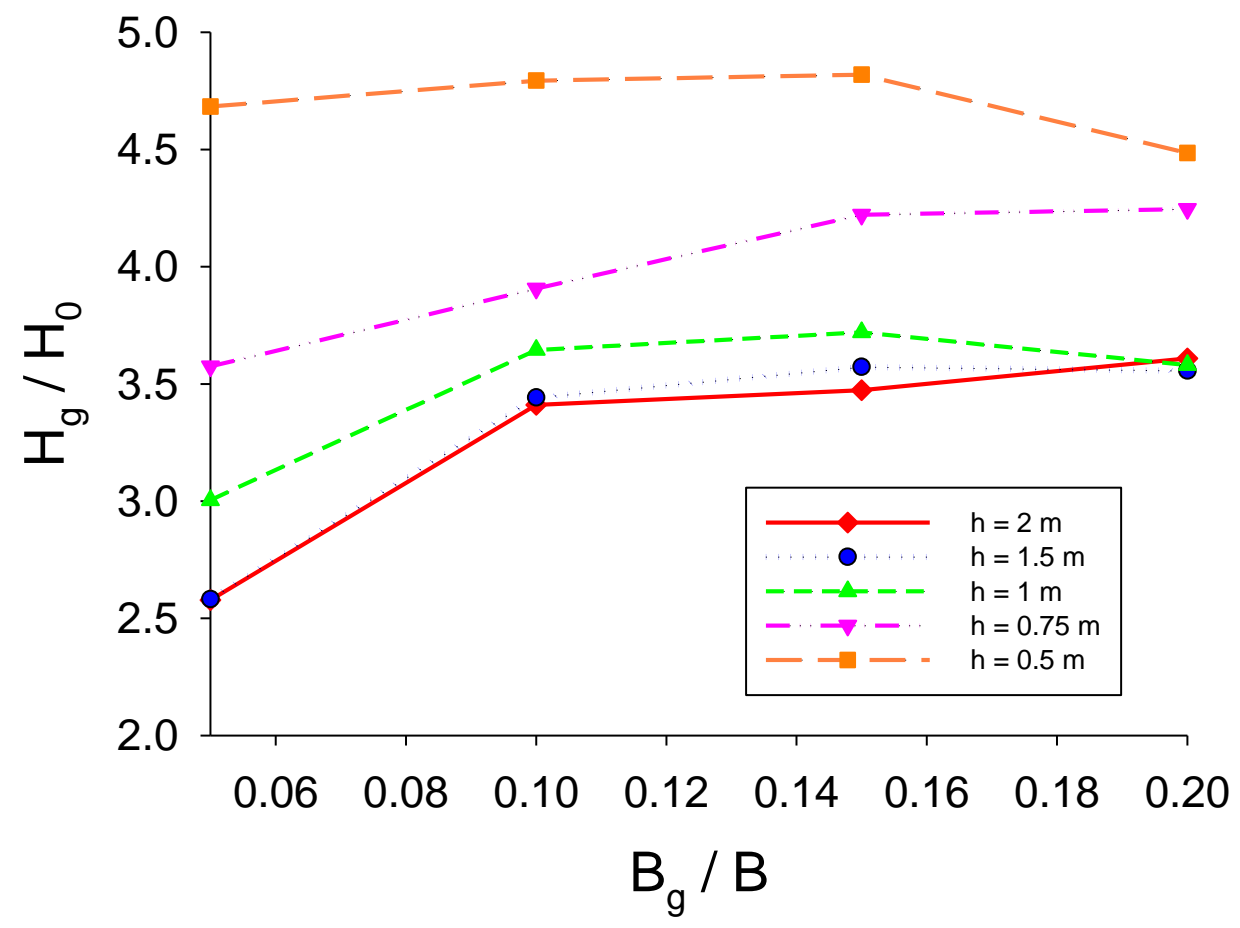

Figure 21. Comparison of the non-dimensional wave height as a function of normalized gap width for different water depths at $\mathrm{d}=0.25 \mathrm{~m}$. 


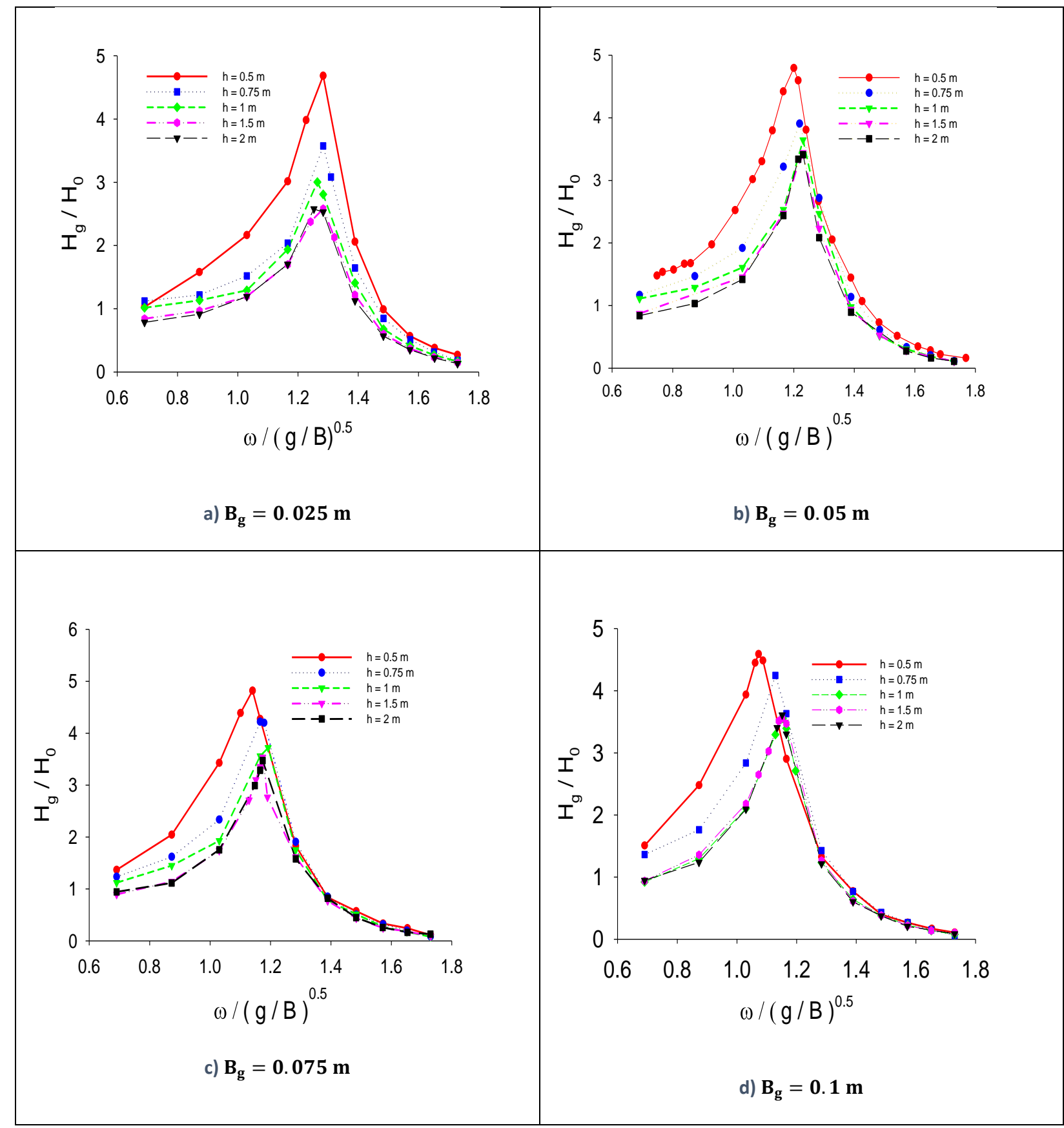

Figure 22. Variation of the non-dimensional wave height as a function of normalized frequency for different water depths and gap widths at $d=0.25 \mathrm{~m}$. 


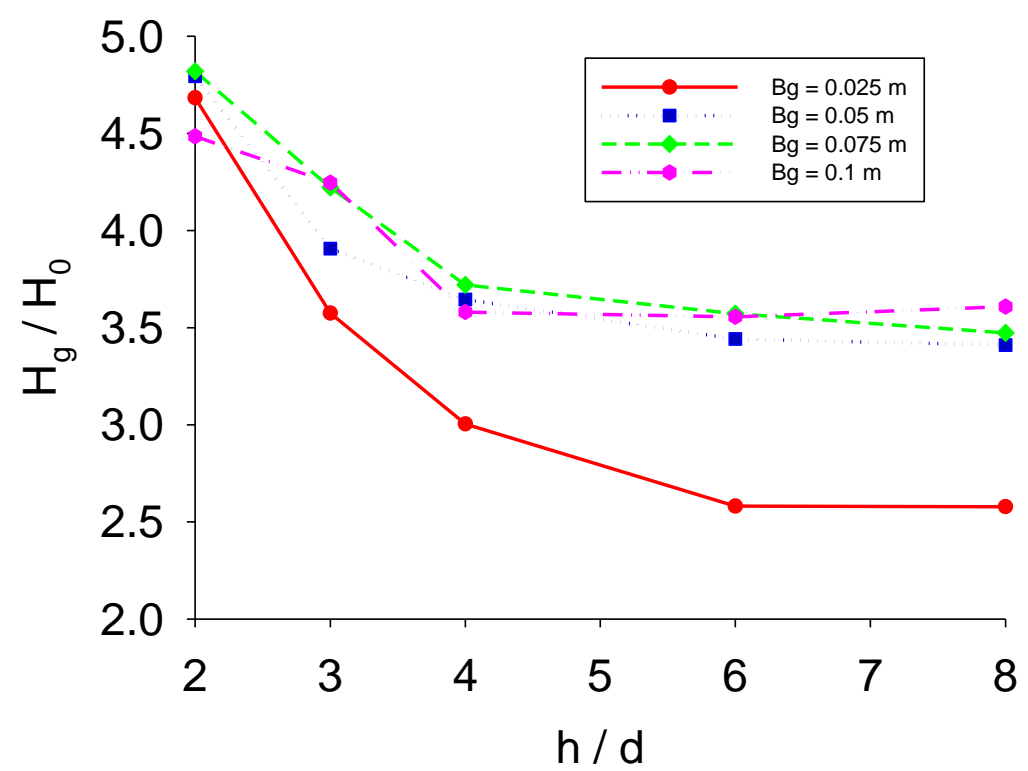

Figure 23. Comparison of resonant wave height as a function of normalized water depth for different gap widths at $\mathrm{d}=0.25 \mathrm{~m}$. 